\title{
ÁRBOLES Y ARBUSTOS INVASORES DE LA PROVINCIA DE CÓRDOBA (ARGENTINA): UNA CONTRIBUCIÓN A LA SISTEMATIZACIÓN DE BASES DE DATOS GLOBALES
}

\author{
MELISA A. GIORGIS ${ }^{1 *}$ y PAULA A. TECCO ${ }^{1}$
}

\begin{abstract}
Resumen: Se presenta la primera base de especies invasoras arbóreas y arbustivas de la Provincia de Córdoba y se analiza en el contexto de las invasiones por leñosas a escala nacional y mundial. Se identificaron 34 especies leñosas exóticas invasoras. La mayoría son originarias de Europa, Asia y Medio Oriente, introducidas como ornamentales. Los ecosistemas más invadidos son los pastizales (39\%) seguido por bosques y matorrales (32\%). La mayoría (59\%) se dispersan por animales (aves) y viento (35\%). A excepción de dos especies, la mayoría son también invasoras en otros lugares del mundo. No obstante, solo la mitad de las invasoras de Córdoba se registraban como invasoras de Sudamérica. Esto último refleja la magnitud en la cual se está subestimando la invasibilidad del continente Sudamericano. Dos especies (Cupressus macrocarpa y Zanthoxylum armatum) se incorporarían como nuevas especies en la base mundial de leñosas invasoras. Se detalla la información faltante en dos bases nacionales, esperando contribuir a la actualización de estos valiosos documentos. Finalmente se listan seis especies nativas de Argentina que estarían invadiendo comunidades vegetales de Córdoba. Esperamos que esta base de datos aporte al desarrollo de estudios básicos y aplicados en la ecología de las invasiones biológicas.
\end{abstract}

Palabras clave: Base de datos, exóticas invasoras, nativas invasoras, invasibilidad, invasividad, plantas leñosas, Sudamérica

Summary: Invasive alien trees and shrubs in Córdoba Province (Argentina): a contribution to the systematization of global bases. We present the first database of invasive alien trees and shrubs of Córdoba Province and analyze it in the context of current national and global databases. We identified 34 invasive woody alien species. Most aliens are from Europe, Asia and Middle East origin, introduced for horticulture reasons. Grasslands are the most invaded ecosystem (39\%), followed by woodlands and shrublands (32\%). Most of the species (59\%) are dispersed by animals (birds) and wind (35\%). Except for two species, most of the species has also been recorded as invaders in other areas of the world. However, only half of them are currently registered as invaders in South America. This might reflect the notably lower research on biological invasions within the continent and the subsequent bias and underestimation of its invasibility. Two species (Cupressus macrocarpa and Zanthoxylum armatum) should be incorporated as new species in the global database. We also refer to some missing information in two major national databases, hoping to contribute with their updating. Finally, we mention six species native to Argentina to be considered as native invaders of Cordoba Province ecosystems. Overall, we expect to provide useful information for the development of basic and applied studies of biological invasions.

Key words: Database, invasive exotic species, invasive native species, invasibility, invasivity, woody plants, South America.

\footnotetext{
1. Instituto Multidisciplinario de Biología Vegetal (IMBIV), CONICET y Facultad de Ciencias Exactas, Físicas y Naturales, Universidad Nacional de Córdoba, Ciudad Universitaria, 5000-Córdoba, Argentina.

melisagiorgis@hotmail.com
} 


\section{INTRODUCCIÓN}

Es ampliamente reconocido que las especies invasoras son uno de los principales problemas ambientales en el mundo(MA2005). Particularmente importante es el impacto que producen los árboles y arbustos invasores sobre la biodiversidad y los servicios de los ecosistemas (por ejemplo; Cronk \& Fuller, 1995; Le Maitre et al., 1996; Holmes \& Cowling 1997; Zavaleta et al., 2000; Jobaggy et al., 2013; Furey et al., 2014; Richardson et al., 2014). A pesar de la relevancia de la problemática y los esfuerzos de la comunidad científica por estudiarla, a la fecha el potencial de generalización o predicción en ecología de invasiones sigue siendo limitado (Prieur-Richard \& Lavorel, 2000; Pyšek et al., 2008). Un importante obstáculo para la formulación de teorías generales de invasiones, es que todavía se desconoce la ecología de numerosas especies invasoras y su impacto en los diversos ecosistemas. El cuerpo teórico de la ecología de invasiones se ha desarrollado principalmente en base a patrones observados en Norteamérica, Europa y Australia, los cuales no necesariamente se corresponden con otras regiones del mundo (Vázquez \& Aragón 2002; Pyšek et al. 2008; Nuñez \& Pauchard, 2010). Esto pone en evidencia la importancia de incrementar las investigaciones en regiones menos estudiadas ya que este tipo de información es la materia prima utilizada en las revisiones y otros trabajos de síntesis.

El sur de Sudamérica es un ejemplo de una región en la cual el conocimiento de la ecología de las especies invasoras y de la invasibilidad de los ecosistemas es incipiente (Vázquez \& Aragón 2002; Pauchard et al. 2004). Recién en las últimas décadas se han incrementado los estudios en los cuales se examina la invasibilidad de algunos ecosistemas, las características de algunas especies invasoras y el impacto de algunas invasoras leñosas (ver artículos citados en Vazquez \& Aragón, 2002; Gutiérrez \& Bernstein, 2014). A pesar de este creciente interés y significativo aporte al desarrollo de ecología de invasiones en la región, todavía se está lejos de los estándares alcanzados en países más desarrollados (Speziale et al., 2012).

Un punto de partida esencial para abordar la problemática de las invasiones tanto a escala local como global, lo constituyen los listados de bases actualizadas de especies invasoras (Rejmánek
\& Richardson, 2013). En Argentina existen contribuciones muy importantes sobre la taxonomía de las especies exóticas, teniendo en cuenta aquí desde el trabajo trascendental de Parodi (1959) y sus posteriores actualizaciones $(1972,1977,1987)$, hasta contribuciones recientes (por ejemplo; Hurrell \& Bazzano, 2003, 2006; Hurrell et al., 2004). Sin embargo, el conocimiento sobre cuáles son las especies invasoras (esto es; aquellas que logran tener poblaciones estables por más de 10 años sin la asistencia del hombre y que además producen descendencia reproductiva a considerables distancias de la planta madre, sensu Richardson \& Rejmánek, 2011) es muy escaso. Un aporte pionero para la Argentina en la temática ha sido el trabajo de recopilación y síntesis coordinado por Zalba y colaboradores; el cual dio origen al primer listado de especies invasoras a nivel nacional (base I3N: www.inbiar.org.ar). Nuestro trabajo espera poder contribuir y complementar estos esfuerzos previos. Específicamente, nos propusimos elaborar una base de datos de las especies invasoras de árboles y arbustos de la Provincia de Córdoba, Argentina. Además del listado de especies, se procuró que la base de datos incluyera información de la especie (taxonómica, lugar de origen, modo de dispersión), una síntesis en cuanto al tipo de ecosistemas que invade en el centro de Argentina, la existencia de antecedentes de invasión en otras partes del mundo, y motivos por los cuales fueron introducidas. Finalmente, se analizó el listado de leñosas invasoras de la Provincia de Córdoba con respecto a los principales listados nacionales e internacionales preexistentes, con el objetivo de identificar aquellas especies invasoras que no hayan sido registradas previamente y aportar a la actualización de las mismas. Esto último tiene relevancia global, ya que la permanente actualización de los listados de invasoras es clave tanto para el desarrollo teórico de la disciplina como para la planificación de estrategia de manejo y conservación de los ecosistemas en cualquier parte del mundo (Rejmánek \& Richardson, 2013).

\section{Materiales y Métodos}

El área de estudio abarcada por esta revisión de leñosas invasoras corresponde a la Provincia de Córdoba (Latitud $29^{\circ} 39^{\prime}-35^{\circ} 06^{\prime}$ S, Longitud 
$61^{\circ} 54^{\prime}-65^{\circ} 47^{\prime} \mathrm{W}$ ), que incluye las provincias fitogeográficas Chaqueña, Espinal y Pampeana (Cabrera, 1976). Hoy en día la primera se encuentra representada por bosques secundarios, arbustales degradados, pastizales y comunidades leñosas dominadas por especies invasoras (Zak et al., 2004; Gavier \& Bucher, 2004; Hoyos et al., 2010; Giorgis et al., 2011; Gavier-Pizarro et al., 2012). El Espinal por su parte, se encuentra casi en su totalidad transformado a monocultivos de soja u otros, quedando pequeños relictos de bosque secundario con distinto grado de alteración e invasión de especies exóticas (Zak et al. 2004; Clausen et al., 2008; Lewis et al., 2009; Noy-meir et al., 2012). Finalmente la vegetación de la Provincia Fitogeográfica Pampeana también ha devenido en grandes extensiones de cultivos, con algunos relictos de comunidades de pastizales inundables o bajos salobres (Brown et al., 2006). Al igual que el Espinal y el Chaco esta región tampoco está exenta de la invasiones por leñosas exóticas (Chaneton et al., 2002; Zalba \& Villamil, 2002).

Para que el listado de invasoras leñosas pueda ser incluido en posteriores actualizaciones de la Base Global de Árboles y Arbustos Invasores a escala mundial (Richardson \& Rejmánek, 2011; Rejmánek \& Richardson, 2013) se siguieron los mismos criterios en cuanto a las definiciones de especies invasoras y la asignación a forma de vida (árbol y arbusto). Es decir, el listado incluye solamente árboles y arbustos invasores (sensu Richardson \& Rejmánek, 2011), y no aquellos que estén solo establecidos en áreas muy disturbadas como bordes de ruta o zonas cultivadas. Como ya mencionamos, este criterio considera que las especies invasoras deben (1) tener poblaciones auto sostenidas por al menos 10 años (ya sea por semillas o reproducción vegetativa, pero sin intervención directa del hombre) y (2) tener un reclutamiento de renovales a distancias considerables de las plantas madres, garantizando el potencial de expansión sobre grandes áreas. Esta definición no posee connotaciones de impacto (ver Richardson et al., 2011; p. 415). Con respecto a la forma de vida, el protocolo de Richardson \& Rejmánek (2011) asigna como árboles aquellas plantas leñosas con un único tronco principal del cual surgen numerosas ramas secundarias claramente separadas del suelo y con una altura superior a los 3 metros. Por su parte, las especies leñosas de menor estatura y/o con múltiples ramificaciones en la base son asignadas a la categoría de arbustos (Richardson \& Rejmánek, 2011).

También de acuerdo a lo discutido en el trabajo de Richardson \& Rejmánek (2011) se procuró identificar aquellas especies originarias de otras regiones fitogeográficas del país que fueron introducidas en la Provincia de Córdoba por el hombre y posteriormente se naturalizaron e invadieron comunidades naturales. Así por ejemplo: Manihot grahamii nativa del noreste de Argentina, aparece como nativa invasora de la Provincia de Córdoba en este trabajo. Seis especies fueron incluidas con este criterio. No obstante, no se incluyeron en las figuras que integran la información de la base excepto expresa indicación en la leyenda. Es decir, se mantuvo siempre la diferenciación entre exóticas invasoras y nativas invasoras.

Para construir la lista de especies arbóreas y nativas de la Provincia de Córdoba nos basamos principalmente en los datos de dos Tesis doctorales con un fuerte componente florístico y énfasis en las invasiones biológicas (Tecco, 2006; Giorgis, 2011). Esta información fue además complementada con una profunda revisión y síntesis de los trabajos publicados sobre invasiones biológicas en la provincia y el país; incluyendo artículos publicados en revistas científicas, catálogos de plantas vasculares y libros botánicos. La revisión bibliográfica se realizó con dos buscadores académicos: Scopus ${ }^{\circledR}$ y SciELO (Scientific Electronic Library Online). En Scopus ${ }^{\circledR}$ la búsqueda se hizo ingresando la siguiente combinación de palabras en inglés: ALL(("Argentina" OR "Cordoba") AND ("tree invasion*" OR "shrub invasion*" OR "woody invasion*" OR "alien shrub*" OR "alien tree*" OR "woody alien*" OR "exotic tree*" OR "exotic shrub*")).

En SciELO se ingresaron las siguientes combinaciones de palabras en castellano: (Argentina) OR (Córdoba) OR (leñosa invasora) OR (arbusto invasor) OR (árbol invasor) OR (arbusto exótico) OR (árbol exótico) OR (leñosa exótica) OR (invasión leñosa).

El criterio de selección en ambos buscadores académicos fue maximizar la probabilidad de encontrar publicaciones sobre invasiones en Argentina (inglés, castellano y portugués) y 
reducir así la probabilidad de pasar por alto algún antecedente de interés para el trabajo. Las búsquedas en Scopus y SciELO fueron realizadas el 29 de Septiembre de 2014 y arrojaron 137 y 115 trabajos, respectivamente. Finalmente una vez armado el listado de especies, se efectuó una nueva búsqueda para cada una de las especies con el buscador académico de Google (scholar. google), ingresando el nombre de la especie más la palabra Argentina). Debido a su naturaleza más inclusiva de Google Académico (Beckmann \& von Wehrden, 2012), esta búsqueda complementaria, permitió encontrar 82 estudios que no habían sido seleccionados en las búsquedas anteriores.

La fuente bibliográfica que sustenta la inclusión de cada una de las especies como invasora de la provincia se encuentra detallada en la base de datos (Apéndice 1). Se incluye además una última columna destacando otras publicaciones realizadas con dichas especies en Argentina. De las 34 especies, cuatro (Acacia dealbata, Bauhinia forficata, Maclura pomifera y Robinia seudoacacia) no disponen de un respaldo bibliográfico para su cita como invasoras de la Provincia de Córdoba. Es decir, fueron incluidas por relevamientos (no publicados) de las autoras.

Una vez confeccionado el listado de especies, se procedió a sintetizar la información de acuerdo a los datos disponibles para cada especie. Con esto se elaboró la base de datos de invasoras de la Provincia en la cual se detalla para cada especie (Apéndice 1): clase superior, orden, familia, nombre completo, forma de vida, lugar de origen, lugares del mundo donde está presente, usos (o razones de su introducción) y forma de dispersión y principales ecosistemas o ambientes invadidos dentro de la Provincia de Córdoba (bosques y matorrales, pastizales, bordes de ríos $\mathrm{y}$ todos estos ambientes). Para la nomenclatura y clasificación taxonómica seguimos la base global publicada por Rejmánek \& Richardson (2013). El lugar de origen, otras regiones del mundo invadidas y tipos de uso, también se basa principalmente en los antecedentes de dicha base global. Con respecto a los principales ecosistemas o ambientes invadidos dentro de la Provincia de Córdoba, la información de esta columna se basa en los datos de las Tesis doctorales de Tecco (2006) y Giorgis (2011), y en las publicaciones de Arana \& Orrego (2004) y Natale et al. $(2008,2013)$. No obstante, cabe aclarar que la información sintetizada está condicionada por la disponibilidad de relevamientos florísticos en la Provincia. Es decir, no se descarta que las especies puedan invadir otros ambientes. Las otras regiones del mundo que están siendo invadidas por las mismas especies que Córdoba (sensu Rejmánek \& Richardson, 2013) fueron incluidas como parte de la base de datos, para ofrecer una descripción del grado de expansión a escala global de cada una de las especies. Las regiones del mundo consideradas fueron: África (sin Sudáfrica); Asia; Australia; Centro América; Europa; Islas del Atlántico; Islas del Caribe; Islas del océano Indico; Islas del Pacífico; Medio Oriente; Norteamérica; Nueva Zelanda; Sudáfrica y Sudamérica.

Finalmente, contrastamos la lista de especies seleccionadas con los listados preexistentes nacionales: (1) base I3N (www.inbiar.org. ar, Invasive alien species database, Argentina Universidad Nacional del Sur, Bahía Blanca) en desarrollo desde el año 2010; y (2) con el Catálogo de las Plantas Vasculares del Conosur (Zuloaga et al., 2008) y sus actualizaciones on-line (www. darwin.edu.ar). En este caso corroboramos si las invasoras estaban registradas para la Provincia de Córdoba, para otras provincias de Argentina y/o para algún otro país del cono sur. Además se evaluó bajo qué estatus estaba asignada (ej, introducida, naturalizada, adventicia, invasora). Por último, comparamos nuestro listado con (3) la base de árboles y arbustos invasores del mundo publicada por Richardson \& Rejmanek (2011) recientemente actualizada (Rejmanek \& Richardson, 2013). Es importante aclarar que para la comparación con las dos bases de Argentina se excluyeron las especies originarias de otras regiones fitogeográficas del país e identificadas como nativas invasoras en Córdoba (ver resultados).

\section{Analisis de datos}

Se evaluaron las diferencias entre árboles y arbustos invasores en función del tipo de ambiente que están invadiendo (bosques y matorrales, pastizales, bordes de ríos y todos estos ambientes) y el modo de dispersión (Anemócoras, Autócoras, Hidrócoras y Zoócoras). Para ello se realizaron test G seguido de comparación de medias utilizando el método de Benjamini \& Hochberg (1995). Para los análisis se usó el paquete "RVAideMemoire" en R 2.15.1 (R Development Core Team 2012). 


\section{Resultados}

Del total de artículos revisados, 132 abordaban el estudio de alguna leñosa invasora en Argentina (excluyendo trabajos en plantaciones exóticas) y sólo 42 las citaban específicamente como invasora de la Provincia de Córdoba. Esta bibliografía avala y/o complementa los datos sintetizados en el Apéndice 1 (taxonomía, lugar de origen, forma de dispersión, tipo de ambiente que invaden en Córdoba y otras regiones del mundo invadidas). En consecuencia, se incluye como información adicional para cada especie dentro de Argentina.

Se identificaron 34 especies exóticas invasoras de la Provincia de Córdoba, pertenecientes a 16 familias y 26 géneros. De las mismas 20 especies correspondieron a árboles (59\%), 12 a arbustos (35\%) y dos pueden ser alternativamente árboles o arbustos (Tamarix spp). Las principales familias presentes sin distinguir por formas de vida, fueron Rosaceae con 11 especies (32\%), seguidas por Fabaceae con cuatro especies (12\%) y Oleaceae con tres especies (9\%).

Dentro de los 22 árboles (incluyendo los Tamarix spp) se encontraron 14 familias, siendo las más numerosas las Rosaceae y Fabaceae con 3 especies cada una, seguido por Moraceae, Oleaceae, Pinaceae y Tamaricaceae con dos especies cada una. Entre los 12 arbustos encontramos cinco familias ( $\sin$ considerar Tamaricaceae), donde las Rosaceae cuentan con 8 especies (67\% de los arbustos invasores), mientras que las restantes (Fabaceae, Olaceae, Rutaceae y Salicaceae), sólo tienen una especie cada una.

El único género con tres especies fue Cotoneaster y los que se mencionan a continuación tienen dos especies cada uno: Ligustrum, Pinus, Prunus, Pyracantha, Rosa y Tamarix. El resto tienen una sola especie por género.

Además de las 34 especies exóticas invasoras, se identificaron seis especies originarias de otras regiones fitogeográficas del país, descriptas como nativas invasoras en Córdoba. Estas especies son: Bauhinia forficata, Jacaranda mimosifolia, Lantana camara, Manihot grahamii, Phytolacca dioica y Schinus areira.

\section{Origen de las invasoras exóticas}

Las leñosas invasoras de la Provincia de Córdoba fueron en su mayoría originarias de Europa, Asia y
Medio Oriente, mientras que el aporte de otras regiones fue menor (Fig. 1).

\section{Ocurrencia de las invasoras en otras regiones del} mundo

La mayoría de las especies exóticas que invaden los ambientes de Córdoba también se han reportado como invasoras en otras regiones del mundo (Fig. 2). Más de la mitad de las invasoras exóticas se encuentran también en Norteamérica, Sudáfrica y Australia (Fig. 2). De las mismas, las tres especies que registran mayor cantidad de regiones invadidas son: Ailanthus altissima, Robinia pseudoacacia y Melia azederach presentes en otras 11, 8 y 8 regiones, respectivamente (Fig. 3). Sin embargo, Lantana camara (especie nativa del noreste de Argentina) supera a todas las especies con antecedentes que la registran como invasora en 12 de las 13 grandes áreas del planeta detalladas en la Fig 2. Sólo cuatro especies, dos originarias de otras regiones de Argentina y dos de Norte América, todavía no han sido registradas como invasoras en la base mundial (Fig. 3).

\section{Usos (o razones de su introducción)}

El $91 \%$ de las 34 especies fueron introducidas por razones ornamentales. Le siguen en importancia el uso forestal (6 especies) y el control de erosión (5 especies). Es importante destacar que la mayoría de las especies son introducidas por un único motivo (21 especies) en tanto que otras tienen dos (8 especies) o hasta tres (5 especies) utilidades (ver Apéndice 1).

\section{Formas de dispersión de las invasoras}

La zoocoria constituye la forma de dispersión más frecuente $(59 \%)$ de las 34 leñosas exóticas invasoras de la provincia. Le sigue en importancia la anemocoria con un $35 \%$, en tanto que solo una especie tendría autocoria y otra hidrocoria. Al desglosar por formas de vida (Fig. 4) se encontraron diferencias marginalmente significativas en el modo de dispersión de ambas $(\mathrm{G}=5,6103 ; p=0,0605)$. La anemocoria estuvo significativamente más representada en árboles que en arbustos, mientras que para el resto de las formas de dispersión no se encontraron diferencias significativas entre las dos formas de vida.

\section{Ambientes invadidos en la provincia de Córdoba}

Teniendo en cuanta las 31 especies con datos del tipo de ambiente invadido en la Provincia, se 


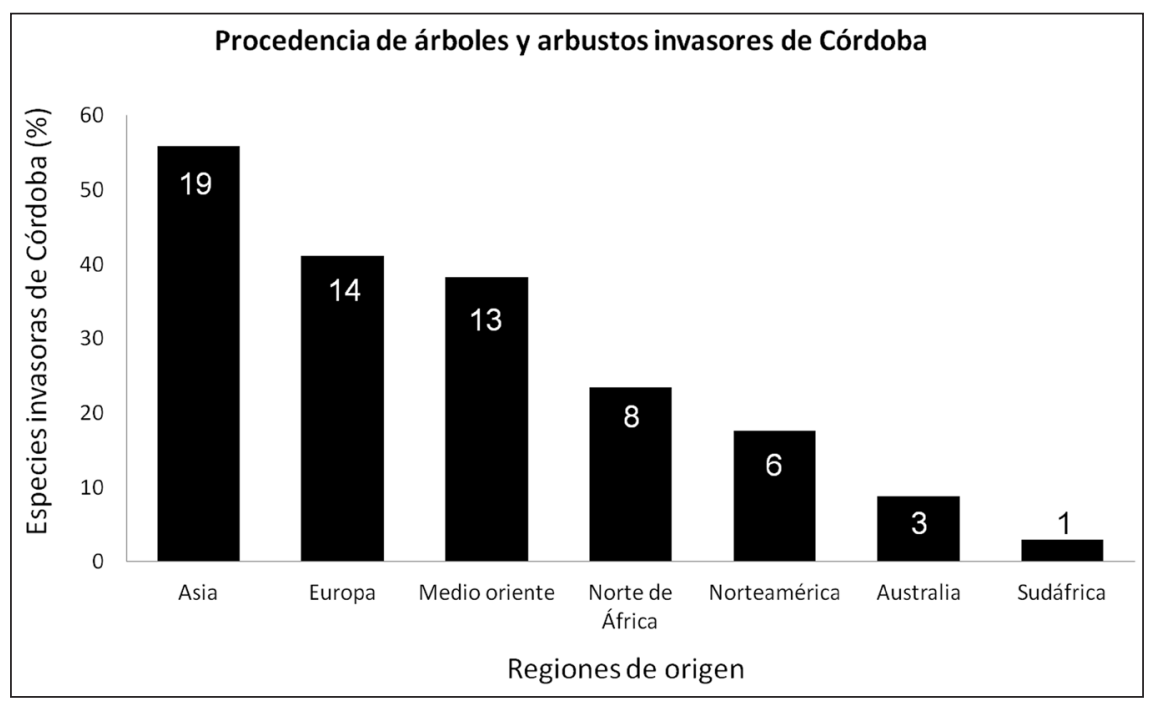

Fig. 1. Origen de los árboles y arbustos exóticos invasores de la Provincia de Córdoba (sensu Rejmánek \& Richardson 2011). En cada barra se grafica el porcentaje de las 34 especies correspondiente a cada region dadora y se indica el número de especies. Algunas especies tienen una distribución original muy amplia (ej. eurasiática) aportando a más de una barra. En consecuencia la suma del número de especies que figura en cada barra supera las 34 invasoras identificadas.

Origin (sensu Rejmánek \& Richardson 2011) of the 34 exotic trees and shrubs identified as invaders in the Cordoba province. Bars show the percentages of these 34 species that belong to each donor region, and the number of species are indicated. Some species have a wide original distribution (e.g. Eurasia) being thus included in more than one bar. Consequently, the sum of the number of species above the bars exceeds the 34 identified invaders.

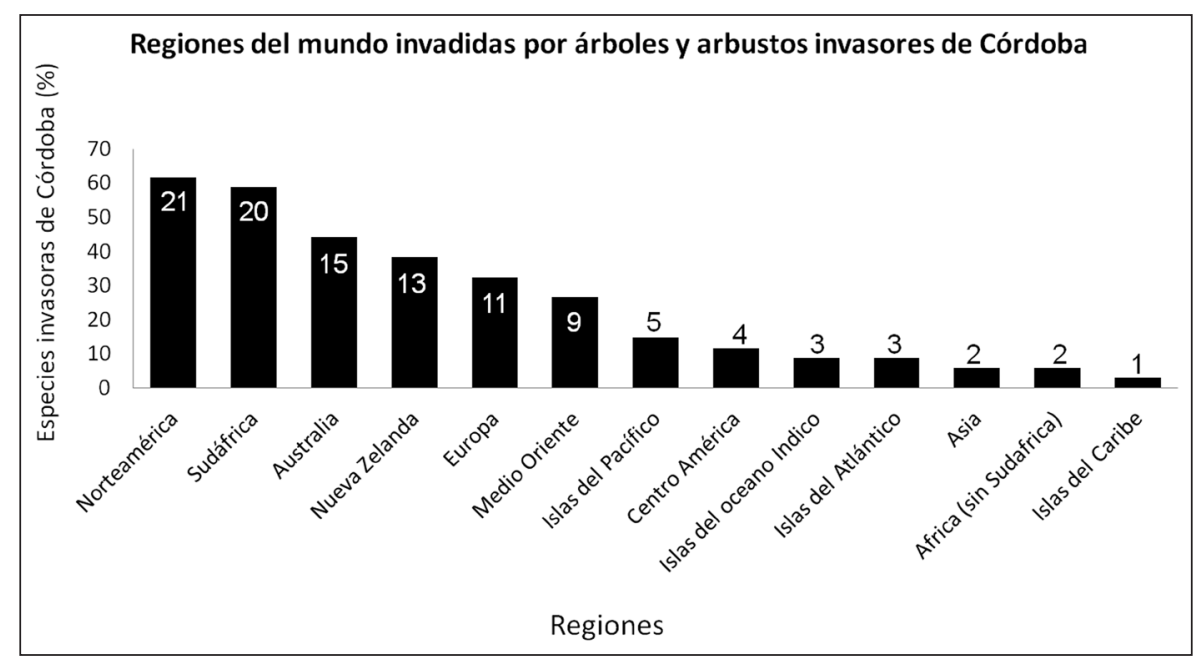

Fig. 2. Otras regiones del mundo en las cuales también están invadiendo algunas las 34 especies exóticas invasoras de la Provincia de Córdoba. En cada barra se grafica el porcentaje de las 34 especies que invaden cada región y se indica el número de especies correspondiente.

Others region in the world that are also invaded by some of the 34 exotic trees and shrubs invading the Cordoba province. In each bar we plot the percentage of the 34 species that invade each region, and indicate the corresponding number of species. 


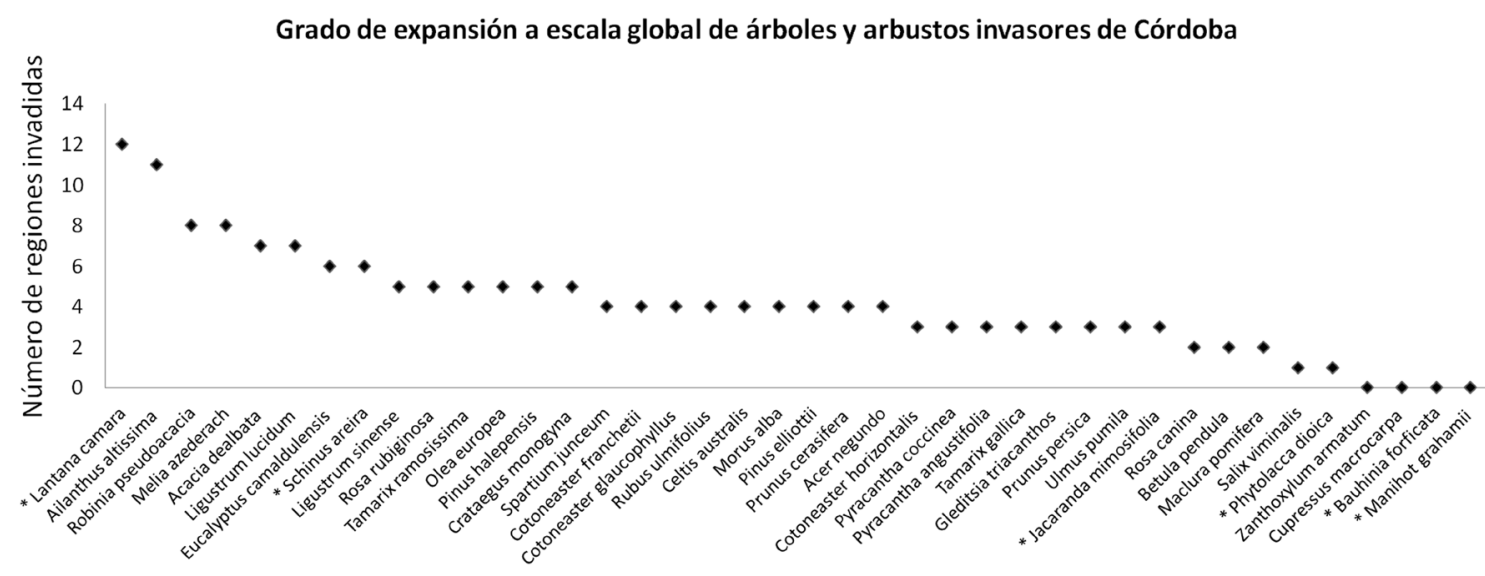

Especies invasoras de la Provincia de Córdoba

Fig. 3. Ranking de las 34 especies de árboles y arbustos exóticos invasores de la provincia de Córdoba en función del número de áreas del mundo donde están invadiendo. Basado en Rejmánek \& Richardson (2013) pero incluyendo la presencia en Sudamérica de las 18 especies exóticas invasoras que no se registraban en esta región según dicha base mundial. Se añaden además, señaladas con un asterisco, a las seis especies nativas invasoras de la Provincia de Córdoba. En este caso se indican otras regiones no sudamericanas en las cuales también se han registrado como invasoras.

Ranking of the 34 exotic trees and shrubs that invade the Cordoba province in relation to the total number of world regions where they have been recorded as invaders. Based on Rejmánek \& Richardson (2013), but adding further 18 exotic species identified in this study as new records of invasive species in South America. We also included and highlighted with an asterisk, native species from other regions of Argentina, considered as native invaders in the Cordoba province. For these species we indicated other regions of the world (excluding South America) were they have been reported as invader.

observa que los pastizales serían los ambientes que concentran el mayor porcentaje de leñosas invasoras de la región (39\%), seguidos por los bosques y matorrales (32\%), en tanto que hasta ahora sólo cuatro especies $(13 \%)$ han sido registradas invadiendo bordes de río y cinco $(16 \%)$ en todos los ambientes. No se encontraron diferencias significativas en los tipos de ambientes invadidos según sean árboles o arbustos $(\mathrm{G}=$ $6,81 ; p=0,146)$. No obstante, se observa una tendencia de mayor representatividad de árboles que arbustos en ambientes de matorrales y bosques (tanto en número como en porcentaje, Fig. 5). Asimismo tanto para árboles como para arbustos se encontró un bajo número de especies invadiendo todos los ambientes considerados (2 y 3 especies respectivamente, Fig. 5).

\section{Nativas invasoras}

Las seis especies originarias de otras regiones de Argentina, actualmente invasoras en la Provincia de Córdoba, han sido en su totalidad introducidas por su valor ornamental. Actualmente se las registra naturalizadas e invadiendo matorrales y bosques de la provincia. Se dispersan por autocoria (Bahuinia forficata y Manihot grahamii), anemocoria (Jacaranda mimosifolia) y ornitocoria (Lantana camara, Phytolacca dioica y Schinus areira). Como se mencionó previamente, algunas de ellas son invasoras en otras regiones del mundo en tanto que otras no poseen antecedentes previos de invasión (Fig. 3).

Integración de la información con otras bases

La mayoría de las especies citadas como invasoras de la provincia estaban comprendidas en alguno de los listados previos nacionales e internacionales. No obstante, la presente revisión sumaría nuevas especies y/o citas en las tres bases.

(1) Para $I 3 N$

El $68 \%$ de las especies exóticas invasoras 


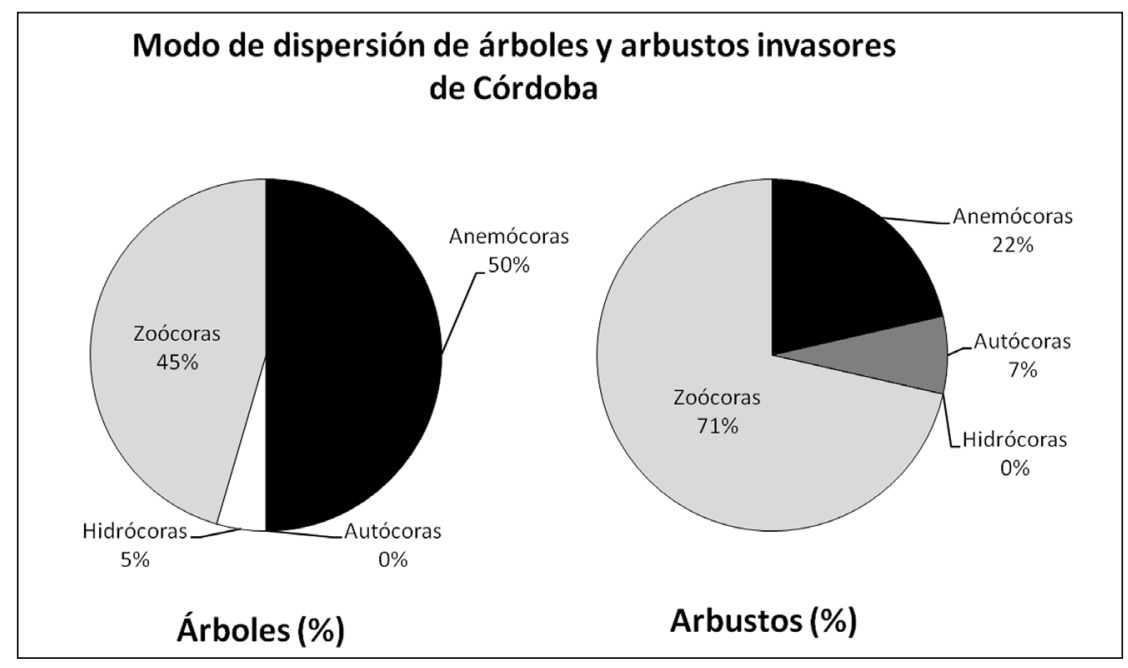

Fig.4. Modo de dispersión de las de las 34 especies de árboles y arbustos exóticos invasores de la Provincia de Córdoba. Se grafican los porcentajes de especies dentro de cada grupo desglosado por árboles (22 especies) y arbustos (14 especies). Las dos especies de Tamarix spp. aportan a ambos grupos debido a que crecen alternativamente como árboles o arbustos. Todas las especies zoócoras son dispersadas por aves, con excepción de una especie arbórea (Gleditsia triacanthos) dispersada por ganado.

Disperal mode of the 34 invasive trees and shrubs Cordoba province. We plot the percentage of species within each dispersal mode broken down into trees (22 species) and shrubs (14 species). Tamarix spp. species contribute to both groups as they grow alternatively as trees or shrubs. All the zoochorous species are dispersal by bird, except for one tree (Gleditsia triacanthos) which is dispersed by livestock.

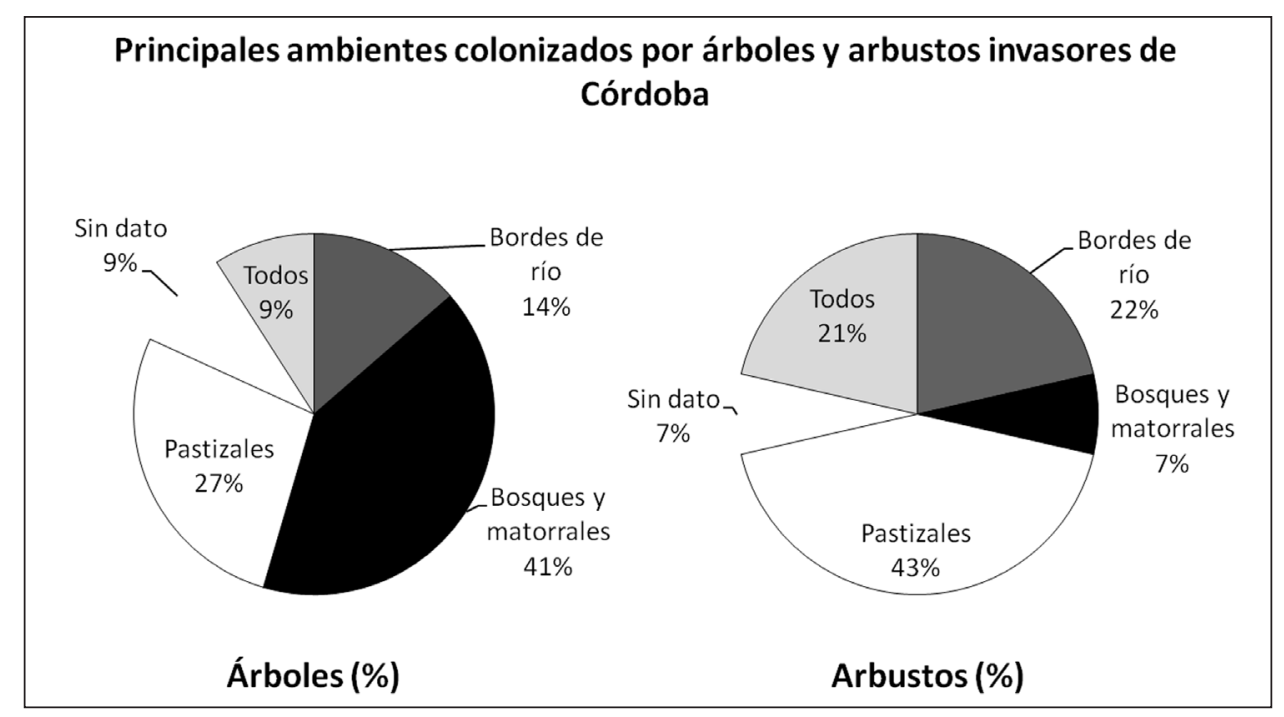

Fig. 5. Tipos de ambientes que invaden los 34 árboles y arbustos exóticos invasores de la Provincia de Córdoba. Se grafican los porcentajes de especies que colonizan cada ambiente desglosado por árboles (22 especies) y arbustos (14 especies). Las dos especies de Tamarix spp se han incluido en ambos grupos debido a que pueden crecer alternativamente como árboles o arbustos.

Types of environment invaded by the 34 invasive trees and shrubs of Cordoba province. We plot the percentage of species colonizing each type of environment broken down into trees (22 species) and shrubs (14 species). Tamarix spp. species contribute to both groups as they grow alternatively as trees or shrubs. 
descriptas en este estudio ya habían sido citadas como invasoras de Argentina en la base del I3N. Restaría incorporar a la misma las siguientes 11 especies exóticas: Ailanthus altissima; Celtis australis; Cotoneaster franchetii; C. glaucophyllus; C. horizontalis; Cupressus macrocarpa; Ligustrum sinense; M. pomífera; Olea europea; Rosa canina y Zanthoxylum armatum.

\section{(2) Para el Catálogo de las Plantas Vasculares del Conosur}

El $62 \%$ de las invasoras exóticas registradas en este trabajo habían sido ya incluidas en la Base del Catálogo (21 de las 34 espcies). No obstante, de las mismas sólo 5 especies se encontraban citadas para Córdoba (Ligustrum lucidum, Melia azederach, Pyracantha angustifolia, Prunus persica y Zanthoxylum armatum), las otras 29 especies serían nuevas citas para la provincia. Asimismo, se sugiere actualizar el estatus de 21 especies (hoy invasoras) ya que 19 están actualmente registradas como adventicias y 2 como introducidas. Finalmente, se sumarían como nuevas citas para Argentina, y en particular para Córdoba, a las siguientes 14 especies: Acacia dealbata, Celtis autralis, Cotoneaster glaucophyllus, Cotoneaster horizontalis, Cupressus macrocarpa, Eucalyptus camaldulensis, Maclura pomifera, Olea europea, Pinus elliotii, Pinus halepensis, Pyracantha coccinea, Robinia pseudoacacia, Tamarix gallica y Ulmus pumilla. En el caso de Acacia dealbata, se encuentra en la base, pero citada como adventicia para Chile, Brasil y Uruguay.

\section{(3) Para la base Global de árboles y arbustos Invasores}

Casi todas las especies exóticas (32 de las 34) que invaden ecosistemas de la Provincia de Córdoba se encontraban incluidas en la base global actualizada de Rejmánek \& Richardson (2013). Sin embargo, solo la mitad (16 especies) se consideraban como invasoras del continente Sudamericano. En consecuencia, en base a nuestros resultados, dos especies serían nuevas especies para incorporar como invasoras a la base mundial (Cupressus macrocarpa y Zanthoxylum armatum), que sumadas a otras 16 actualmente catalogadas como invasoras en otras regiones del globo, se incorporarían como nuevas citas para Sudamérica (18 especies nuevas para Sudamérica en total).

Finalmente, se destaca la importancia y posible incorporación a la base mundial de dos especies nativas de otras regiones fitogeográficas argentinas (Bahuinia forficata y Manihot grahamii) que de acuerdo a Rejmánek \& Richardson (2013) no poseen antecedentes de invasión en otras regiones del mundo.

\section{Discusión}

Frente al irreversible incremento de especies invasoras en todos los ambientes del mundo (Davis, 2006) y a la necesidad de incrementar los antecedentes de invasiones en regiones menos estudiadas como Sudamérica (Pyšek et al., 2008), creemos que esta base de datos de leñosas invasoras de la provincia de Córdoba, constituye una contribución de relevancia tanto a escala local como global. A escala local, provee información de base para desarrollar proyectos de investigación que profundicen en aspectos relacionados con la invasividad de las especies (ej. estrategias de dispersión) y su impacto diferencial en los distintos ambientes invadidos (ej. en ciclado de nutrientes, recursos hídricos, etc.), aportando al cuerpo teórico de la disciplina. Pero además, contribuye a la actualización de un importante catálogo de plantas vasculares y una base online de invasoras del país, ambos documentos de consulta permanente para investigadores y estudiantes. Finalmente, el listado y las descripciones asociadas a cada especie, constituye una herramienta de gran utilidad para planificar estrategias de manejo en la región. A escala global, provee nuevos registros a la base mundial de invasoras leñosas (Rejmánek \& Richardson, 2013) y pone de manifiesto que en Sudamérica la invasión por leñosas está siendo subestimada por falta de registros.

Entre los árboles invasores de Córdoba no hay una clara dominancia de una familia en particular, mientras que sí se observa para los arbustos. Estos últimos pertenecen en su mayoría (el 62\% de los arbustos) a la familia Rosaceae, en consonancia con los resultados encontrado en otros lugares del mundo (Rejmánek, 2014). Este dominio de rosáceas arbustivas invasoras contrasta con la flora nativa de la región, en la cual la representatividad de arbustos de esta familia es muy baja (Giorgis et al. 2011). Por otra parte, Fabaceae constituye una de las familias más representativas de la flora nativa (Zuloaga et 
al. 2008; Giorgis et al. 2011), y es también una familia catalogada como proveedora de numerosas invasoras a nivel mundial (Rejmánek \& Richardson, 2013). Sin embargo, en Córdoba solo se registraron cuatro especies invasoras de esta familia. Por lo pronto, el árbol Gleditsia triacanthos (Fabaceae), es la invasora más frecuente de ambientes invadidos del Chaco Serrano de Córdoba (Giorgis et al. 2011), donde afecta la composición florística y procesos asociados al ciclado de nutrientes (Furey et al., 2014).

En contraste con los patrones descriptos en la base global de invasoras, se registraron para Córdoba sólo dos especies invasoras de la familia Pinaceae (P. elliotii, P. halepensis). La primera fue y continua siendo plantada por su valor forestal en la región en tanto que la segunda es ornamental (Hurrell \& Bazzano, 2006). Dado que otras Pinaceae, también son utilizadas como ornamentales y forestales (ej. Pinus taeda, Pinus radiata, Pinus ponderosa, Pinus patula, Pinus pinaster, http:// cordobaforestal.blogspot.com.ar/p/informaciontecnica.html) es probable que esta familia, que aporta tantas invasoras en otras partes del mundo (Rejmánek \& Richardson, 2013), también aumente su representatividad en esta Provincia en un futuro.

\section{Origen de las invasoras}

La mayoría de las especies invasoras de esta región son originarias del viejo mundo (Fig. 1). Esto está relacionado, como sugiriera Speziale et al., (2012) con el predominio de inmigrantes Europeos en Argentina, que ha condicionado la valoración de especies estrictamente europeas y de otras (asiáticas o de Medio Oriente) previamente introducidas en Europa como ornamentales. En este sentido, es interesante destacar que una región como Australia que aportan más de 100 invasoras al resto del mundo (Rejmánek \& Richardson, 2013) y comparte aspectos climáticos y fisonómicos con el centro de Argentina, no sería todavía una importante fuente de invasoras a nuestra Provincia. Por el momento Eucalyptus camaldulensis y Acacia dealbata serían las únicas invasora en la provincia de Córdoba de origen Australiano. Es muy probable que solo sea el resultado de un menor intercambio con dicho continente. Dado que el principio de precaución es la estrategia más efectiva y de menor costo (Mack et al., 2000), sería promisorio el desarrollo y aplicación de una legislación vinculada a la problemática de las invasiones biológicas, en la cual por ejemplo se restrinja la introducción al país de especies leñosas de origen australiano. En particular aquellas catalogadas en la base de Rejmánek \& Richardson (2013) como invasoras de otros ecosistemas Sudamericanos.

Ocurrencia de las invasoras en otras regiones del mundo

La existencia de antecedentes de invasión en otras regiones, ha sido propuesta como una información muy relevante del potencial invasor (o invasividad) de una especie (Rejmánek, 2000). En este sentido, una de las especies invasoras nativas y una de las exóticas incluidas en esta base de Córdoba, están catalogadas como invasoras en más de 10 regiones del mundo (Lantana camara y Ailanthus altissima). Sin embargo, la cantidad de regiones invadidas a escala mundial no se correspondería en todos los casos con su agresividad dentro de una región invadida en particular. En el caso de la Provincia de Córdoba, un relevamiento florístico exhaustivo de las comunidades vegetales del Chaco Serrano de Córdoba, abarcando bosques en distintos estadios sucesionales, arbustales degradados y pastizales, detectó como principales invasoras a Gleditsia triacanthos y Ligustrum lucidum, seguidas por Morus alba y Pyracantha angustifolia (por más detalles ver Giorgis et al., 2011). Esto no se corresponde con lo esperado según el ranking mostrado en la figura 3. Por ejemplo, la dominancia de Gleditsia triacanthos en esta y otras provincias del país (Cannas et al., 2003; Mazia et al., 2010; Giorgis et al., 2011) contrasta con el bajo número de regiones (2) en la cual se la considera invasora a escala mundial (Fig. 3). Lo propio ocurre con las otras tres especies. Esto puede deberse en parte al sesgo asociado a la existencia de regiones más estudiadas que otras (Pyšek et al., 2008), pero también a la idiosincrasia de cada invasión (Pyšek \& Richardson, 2007).

\section{Usos (o razones de su introducción)}

La mayoría de las especies fueron introducidas por razones ornamentales, al igual que lo encontrado por Rejmánek \& Richardson, (2013). Como ya se mencionó, esto es un patrón recurrente que refleja, en el caso particular de Argentina, la impronta de nuestra historia de inmigración (Speziale et al., 2012). Lejos de juzgar eventos pasados, 
estrechamente ligados al desarraigo, creemos necesario revisar los hábitos contemporáneos. Actualmente sigue predominando en los viveros la demanda de especies exóticas como ornamentales. La difusión y educación sobre las ventajas de utilizar especies nativas como ornamentales sería un punto de partida para cambiar la percepción de la sociedad. Pero además debería complementarse con incentivos a su producción (en viveros) y respaldarse con estudios que analicen las opciones nativas que respondan a las distintas necesidades urbanas (ej., belleza, sombra, crecimiento rápido, raíces que no rompan veredas, fustes altos, etc.).

El uso de especies exóticas como forestales es más discutido. Al beneficio económico que implica la producción de madera de pino se le contrapone el costo sobre el rendimiento hídrico del sistema (Farley et al., 2005; Licata et al. 2008; Jobbagy et al., 2013). Esta situación es particularmente importante en ecosistemas donde las precipitaciones son menores a $1200 \mathrm{~mm}$ anuales; condición en la cual se ha registrado una reducción del $44 \%$ y $31 \%$ del caudal de los ríos cuando las plantaciones reemplazan pastizales y matorrales, respectivamente (Farley et al., 2005). En la Provincia de Córdoba constituye un conflicto evidente, considerando que las plantaciones de pinos se localizan en su mayoría en las Sierras Grandes de Córdoba, principal cabecera de cuenca de la Provincia.

Modos de dispersión y tipo de ambientes invadidos de las especies leñosas invasoras de Córdoba

En consonancia con otras bases mundiales el principal modo de dispersión de las plantas invasoras es a través de animales (aves) y en menor medida por el viento (Pyšek \& Richardson, 2007; Reichard, 2011). Esto puede responder, como destacara Richardson \& Rejmánek (2011) a que la mayoría de las especies ornamentales se caracterizan por atributos como profusa fructificación con vistosos frutos carnosos atractivos para un amplio elenco de dispersores, que por añadidura les confiere gran invasividad (Reichard, 2011; Pyšek et al., 2014). En Córdoba, varias de estas invasoras fructifican en otoño-invierno, a diferencia de las nativas que lo hacen en la estación cálida (Gurvich et al., 2005; Tecco et al., 2006, 2013). Cabe preguntarse (y estudiar) cuál será el impacto de todas estas leñosas invasoras en las comunidades de aves de la región y en la compleja dinámica de interacciones. Por ejemplo, en otras regiones se ha observado el desarrollo de complejos de dispersión multiespecíficos incorporando plantas y dispersores exóticos que facilitan el reclutamiento de más aves y plantas exóticas (Buckley et al., 2006).

Estudios previos han vinculado el tipo de dispersión de una especie con el tipo de ambiente que invade (Cronk \& Fuller, 1995). Así, las especies anemócoras serían las invasoras típicas de ambientes abiertos (pastizales) en tanto que la zoocorias predominaría entre invasoras de comunidades cerradas (bosques y matorrales). Sin embargo, esta segregación no se observa entre las leñosas invasoras de Córdoba. Tanto la zoocoria como anemocoria estuvieron ampliamente representadas en comunidades abiertas y cerradas de la provincia (Fig. 6). En todo caso se destacó la zoocoria como la estrategia de dispersión más efectiva para colonizar simultáneamente todos los tipos de ambientes considerados. Estudios específicos y monitoreos periódicos serán necesarios para evaluar este patrón.

\section{Nativas invasoras}

Para conocer los cambios en la distribución de especies nativas se requieren estudios detallados tanto palinológicos como búsquedas bibliográficas y revisiones profundas en herbarios nacionales e internacionales. Como hasta el momento no hay estudios al respecto, resulta muy difícil establecer un límite para clasificar como invasora a una especie originaria de eco-regiones vecinas (ej. del pedemonte de las Yungas). En este trabajo, sugerimos que seis especies sudamericanas pueden considerarse invasoras nativas de los bosques y matorrales de la Provincia de Córdoba. Por ejemplo, catalogamos a Schinus areira (Sinónimo de S. molle L.) como invasora del bosque serrano de Córdoba, basadas en el contraste de trabajos florísticos históricos de la provincia (Kurtz, 1904, Sayago, 1969) con registros actuales (Giorgis, 2011) y revisiones bibliográficas. Sin embargo, dado que este criterio es cuestionable y no está avalado por datos paleobotánicos, las seis especies se presentan claramente diferenciadas de las especies exóticas (i.e., originarias de otros continentes) en la base de datos. El estudio de aspectos relacionados con el impacto de estas "invasoras nativas" y su comparación con los impactos de las invasoras exóticas, aportarían 


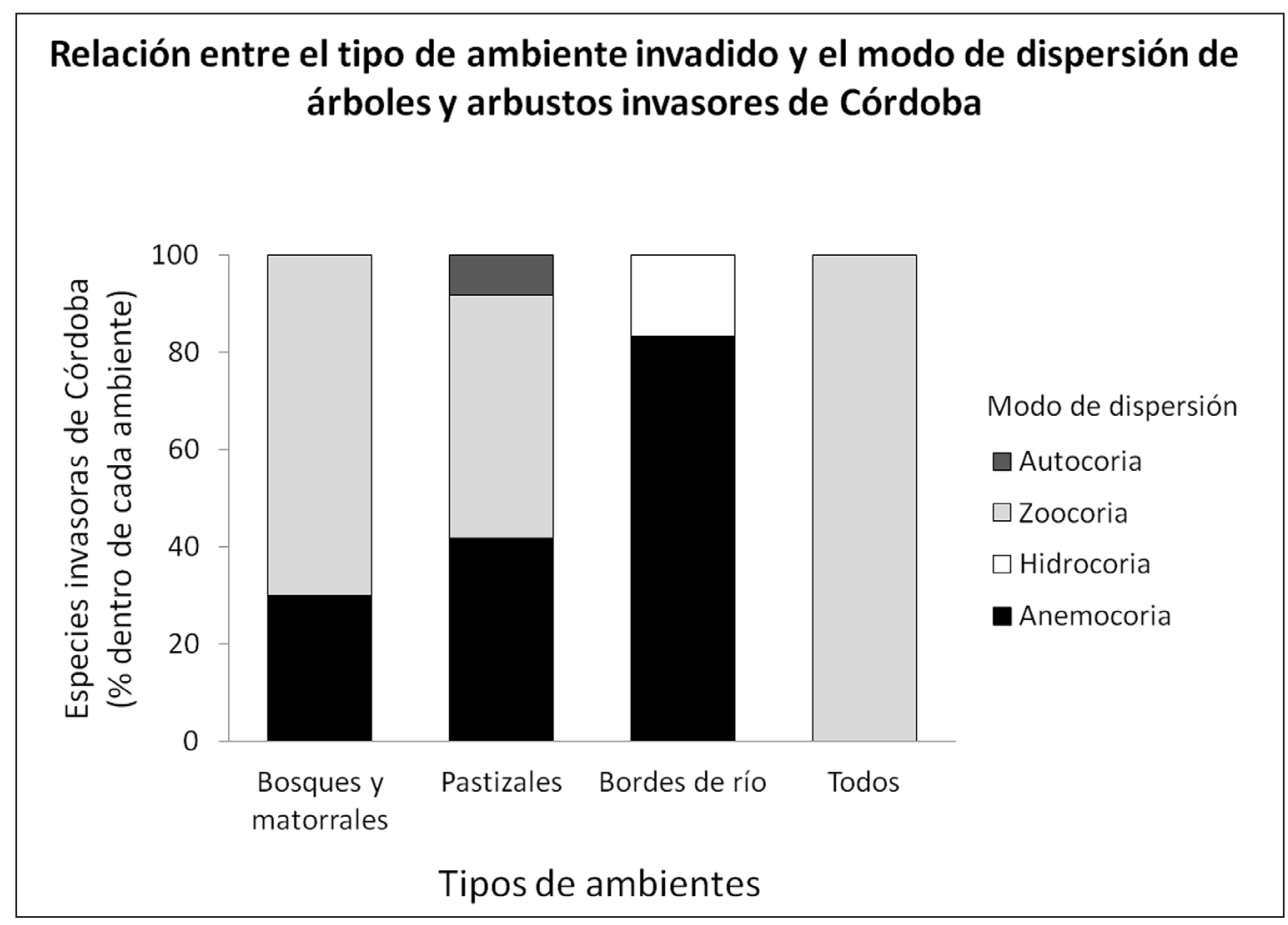

Fig. 6. Relación entre el modo de dispersión de los árboles y arbustos exóticos invasores de la Provincia de Córdoba y el tipo de ambientes que invaden. Se grafica, para cada tipo de ambiente, la proporción de especies correspondiente a cada modo de dispersión. Se excluyeron las tres especies para las cuales no se dispone de datos del ambiente invadido.

Relationship between the dispersal mode and the type of invaded environment for the 34 invasive trees and shrubs of Cordoba province. We plot the proportion of species corresponding to each dispersal mode within the different environments. Three species were due to lack of data on the types of invaded environments.

significativamente al debate de su inclusión (o no) en proyectos de restauración de ecosistemas degradados (ej, Diaz et al., 2011) y en programas de control de invasoras. Independientemente del resultado de estas comparaciones, destacamos los casos de Manihot grahamii y Bauhinia forficata, originarias de regiones más húmedas y cálidas del noreste argentino. Ninguna de las dos ha sido reportada como invasora en otra parte del mundo, a pesar de poseer congéneres que sí lo son (Rejmánek \& Richardson, 2013). Su estatus de invasora en los ecosistemas de la Provincia de Córdoba alertan sobre la importancia de evitar su introducción en otros lugares del mundo.

\section{Invasibilidad de Sudamérica}

De acuerdo a la última actualización de la base mundial, Sudamérica es catalogada como una región proveedora de gran número de invasoras a otras regiones del mundo (más de $100 \mathrm{spp}$ ), pero con una menor invasibilidad que otras regiones. Específicamente, se cita a Sudamérica como susceptible a la mitad de las invasoras (111) registradas para otras regiones como Norteamérica (212), Australia (203) o Sudáfrica (178) (Rejmánek \& Richardson, 2013). Sin embargo, la inclusión de una sola provincia política de Argentina aporta 18 nuevos registros para todo un continente, lo cual revela la necesidad de aumentar los esfuerzos científicos destinados al estudio de las invasiones biológicas en Sudamérica (Richardson et al., 2014). No encontramos sustento histórico ni ecológico que avale que todo un continente que abarca más de siete biomas (Selva tropical y subtropical, Sabana, matorral mediterraneo, bosque templado, pastizal templado, bioma de montaña y desierto; Archibold, 1995) posea tal diferencia de invasibilidad con respecto a los demás continentes. Probablemente 
sea tan solo el reflejo de la escasez de estudios publicados sobre invasoras en Sudamérica (Speziale et al., 2012). En suma, este trabajo aporta más evidencia para la inclusión de Sudamérica como un nuevo hotspot de invasión (sensu Richardson et al., 2014), como ya lo son Sudáfrica, Australia y Nueva Zelanda.

Por último quisiéramos remarcar que esta contribución incluye registros publicados hasta fines del 2014. En consecuencia, como toda base de datos, deberá ser actualizada regularmente (Rejmánek \& Richardson, 2013). Dado que las especies invasoras identificadas seguirán expandiendo sus áreas de distribución, y que las actividades humanas continúan propiciando la llegada y establecimiento de nuevas especies, esperamos que la comunidad científica y los tomadores de decisiones incrementen los esfuerzos destinado a su estudio, registro, publicación, prevención y control.

\section{Agradecimientos}

Este trabajo fue financiado por SECYT, Universidad de Córdoba, FONCyT (PICT 953) y el Inter-American Institute for Global Change Research (IAI) CRNII-2005. Los autores agradecen al IMBIV, al Consejo Nacional de Investigaciones Científicas y Técnicas (CONICET) y a la Universidad Nacional de Córdoba por las facilidades brindadas para llevar a cabo esta investigación. A Gustavo Delucchi por ayuda en la identificación taxonómica de algunas especies. Y finalmente a Ana M. Cingolani y a dos revisores anómimos por sus valiosos comentarios y sugerencias. Las autoras son investigadoras del CONICET y docentes de la UNC.

\section{Bibliografía}

ARCHIBOLD, O. W. 1995. Ecology of world vegetation. Chapman and Hall. Londres.

BECKMANN, M. \& H. VON WEHRDEN. 2012. Where you search is what you get: Literature mining - Google Scholar vs ISI using a dataset from a literature search in vegetation science. J. Veg. Sci. 23: 1197-1199.
BENJAMINI, Y. \& Y. HOCHBERG. 1995. Controlling the false discovery rate: a practical and powerful approach to multiple testing. J. Roy. Stat. Soc. B 57: 289-300.

BROWN, A., U. MARTÍNEZ ORTIZ, M. ACERBI \& J. CORCUERA. 2006. La Situación Ambiental Argentina. Fundación Vida Silvestre Argentina, Buenos Aires.

BUCKLEY, Y. M., S. ANDERSON, C. P. CATTERALL, R. T. CORLETT, T. ENGEL, C. R. GOSPER, R. NATHAN, D. M. RICHARDSON, M. SETTER, O. SPIEGEL, G. VIVIAN-SMITH, F. A. VOIGT, J. E. S. WEIR \& D. A. WESTCOTT. 2006. Management of plant invasions mediated by frugivore interactions. J. Appl. Ecol. 43: 848-857.

CABRERA, A. L. 1976. Regiones fitogeográficas argentinas. Enciclopedia Argentina de agricultura y jardinería. ACME S.A.I.C., Buenos Aires.

CANNAS, S. A., D. E. MARCO \& S. A. PÁEZ. 2003. Modelling biological invasions: species traits, species interactions, and habitat heterogeneity. Math. Biosci. 183:93-110.

CHANETON, E. J., S.B. PERELMAN, M. OMACINI \& R. J. C. LEÓN. 2002. Grazing, environmental heterogeneity, and alien plant invasions in temperate Pampa grasslands. Biol. Invasions 4: 7-24.

CLAUSEN, A. M., M. E., FERRER \& M. B. FORMICA. 2008. Situación de los Recursos Fitogenéticos en la Argentina. II Informe Nacional 1996-2006. INTA, Córdoba.

CRONK, Q. C. B. \& J. L. FULLER. 1995. Plant invaders, the threat to natural ecosystems, 1 st ed. Chapman and Hall, London.

DAVIS M. A. (2006) Invasion biology 1958-2005: the pursuit of science and conservation. In: CADOTTE M. W., S. M. MCMAHON \& T. FUKAMI (eds.), Conceptual ecology and invasion biology, pp. 35-64. Springer, Great Britain.

DÍAZ, S., L. ENRICO, D. CÁCERES, A. CHEBEL, A. M. CINGOLANI, A. CUCHIETTI, D. GURVICH, F. KOPTA, R. KOPTA, J. M. MENNA, J. L. NAVARRO, G. RE, P. A. TECCO, C. URCELAY \& E. VIDALLÉ. 2011. Propuesta de Franjas Forestales de Caminos para Múltiples Beneficios Ecosistémicos en la Provincia de Córdoba. Disponible en: http://www.nucleodiversus.org/ uploads/file/forestaciondecaminos.pdf [Acceso: 5 Agosto 2011].

FUREY,C.,P.A.TECCO, N.PEREZ-HARGUINDEGUY, M. A. GIORGIS \& M. GROSSI. 2014. The importance of native and exotic plant identity and dominance on decomposition patterns in mountain woodlands of central Argentina. Acta Oecol. 54: 13-20.

GAVIER, G. I. \& E.H. BUCHER. 2004. Deforestación 
de las Sierras Chicas de Córdoba (Argentina) en el período 1970-1997. Academia Nacional de Ciencias. Miscelánea $\mathrm{N}^{\mathrm{0}} 101$.

GAVIER-PIZARRO, G. I., T. KUEMMERLE, L. E. HOYOS, S. I. STEWART, C. D. HUEBNER, N. S. KEULER \& V. C. RADELOFF. 2012. Monitoring the invasion of an exotic tree (Ligustrum lucidum) from 1983 to 2006 with 1 Landsat TM/ETM+ satellite data and support vector machines in Córdoba, Argentina. Remote Sensing Environ. 122: 134-145.

GIORGIS, M. A. 2011. Caracterización florística y estructural del Bosque Chaqueño Serrano (Córdoba) en relación a gradientes ambientales y de uso. Tesis doctoral. Universidad Nacional de Córdoba. Argentina.

GIORGIS, M. A., A. M. CINGOLANI, F. CHIARINI, J. CHIAPELLA, G. BARBOZA, L. ARIZA ESPINAR, R. MORERO, D. E. GURVICH, P. A. TECCO, R. SUBILS \& M. CABIDO. 2011. Composición florística del Bosque Chaqueño Serrano de la provincia de Córdoba, Argentina. Kurtziana 36: $9-43$.

GURVICH, D. E., P. A. TECCO \& S. DÍAZ. 2005. Plant invasions in undisturbed ecosystems: The triggering attribute approach. J. Veg. Sci. 16: 723-728.

GUTIÉRREZ, J. L. \& C. BERNSTEIN. 2014. Ecosystem impacts of invasive species. BIOLIEF 2011 - 2nd World Conference on Biological Invasion and Ecosystem Functioning, Mar del Plata, Argentina, 21-24 November 2011. Acta Oecol. 54: 1-138.

HOLMES, P. M. \& R. M. COWLING. 1997. The effects of invasion by Acacia saligna on the guild structure and regeneration. J. Appl. Ecol. 34:317-332.

HOYOS, L. E., G. I. GAVIER-PIZARRO, T. KUEMMERLE, E. H. BUCHER, C. VOLKER, V. C. RADELOFF \& P. A. TECCO. 2010. Invasion of glossy privet (Ligustrum lucidum) and native forest loss in the Sierras Chicas of Córdoba, Argentina. Biol. Invasions 12: 3261-3275.

HURRELL, J. A. \& D. BAZZANO. 2003. Arbustos 1 nativos y exóticos. Biota Rioplatense Volumen VIII. Ed. LOLA. Buenos Aires. Argentina.

HURRELL J. A., \& D. BAZZANO. 2006. Pinos ornamentales y forestales. Plantas de la Argentina. Volumen 2. LOLA. Buenos Aires. Argentina.

HURRELL, J. A., D. BAZZANO \& G. DELUCCHI. 2004. Arbustos 2 nativos y exóticos. Biota Rioplatense. Volumen VIII. Ed. LOLA. Buenos Aires. Argentina.

JOBBÁGY, E. G., A. ACOSTA \& M. D. NOSETtO. 2013. Rendimiento hídrico en cuencas primarias bajo pastizales y plantaciones de pino de las sierras de Córdoba (Argentina). Ecología Austral 23: 87-96.
KURTZ, F. 1904. Flora de Córdoba. In: Río, M. E. \& L. Achával (eds.), Geografía de la Provincia de Córdoba. Vol. 1, pp. 270-343. Compañía Sudamericana de Billetes de Banco, Buenos Aires.

LE MAITRE, D. C., B. W. VAN WILGEN, R. A. CHAPMAN \& D. H. MCKELLY. 1996. Invasive plants and water resources in the Western Cape Province, South Africa: modelling the consequences of a lack of management. J. Appl. Ecol. 33:161-172.

LEWIS, J. P., S. NOETINGER, D. E. PRADO \& I. M. BARBERIS. 2009. Woody vegetation structure and composition of the last relicts of Espinal vegetation in subtropical Argentina. Biodivers. Conserv. 18: 3615-3628.

MA 2005. Millennium Ecosystem Assessment. Ecosystems and Human Well-being: Synthesis. Island Press, Washington, DC, USA.

LICATA, J. A., J. E. GYENGE, M. E. FERNANDEZ, T. M. SCHLICHTER \& B. J. BOND. 2008. Increased water use by ponderosa pine plantations in northwestern Patagonia, Argentina compared with native forest vegetation. Forest Ecol. Manag. 255: 753-764.

MACK, R. N., D. SIMBERLOFF, W. M. LONDSDALE, H. EVANS, M. CLOUT \& F. A. BAZZAZ. 2000. Biotic invasions: causes, epidemiology, global consequences, and control. Ecol. Appl. 103: 689-710.

MAZÍA C. N., E. J. CHANETON, M. MACHERA, A. UCHITEL, M. V. FELER \& C. M. GHERSA. 2010. Antagonistic effects of large- and small-scale disturbances on exotic tree invasion in a native tussock grassland relict. Biol. Invasions 12: 31093122.

NOY-MEIR, I., M. MASCÓ, M. A. GIORGIS, D. E. GURVICH, D. PERAZZOLO \& G. RUIZ. 2012. Estructura y diversidad de dos fragmentos del bosque de Espinal en Córdoba, un ecosistema amenazado. Bol. Soc. Argent. Bot. 47: 119-133.

NUÑEZ, M. \& A. PAUCHARD. 2010. Biological invasions in developing and developed countries: does one model fit all? Biol. Invasions 12: 707-714

ORDONEZ A., I. J. WRIGHT \& H. OLFF. 2010. Functional differences between native and alien species: a global-scale comparison. Funct. Ecol. 24: 1353-1361.

PARODI, L. 1959. Enciclopedia Argentina de Agricultura y Jardinería. Buenos Aires, Argentina, ACME.

PAUCHARD, A. \& P. B. ALABACK. 2004. Influence of elevation, land use, and landscape context on patterns of alien plant invasions along roadsides in protected areas of South- Central Chile. Conserv. Biol. 18: 238-248.

PRIEUR-RICHARD, A. H. \& S. LAVOREL. 2000. Invasions: the perspective of diverse plant communities. Austral Ecol. 25: 1-7. 


\section{A. Giorgis y P. A. Tecco - Leñosas invasoras de Córdoba}

PYŠEK, P., D. M. RICHARDSON, J. PERGL, V. JAROŠÍK, Z. SIXTOVÁ \& E. WEBER. 2008. Geographical and taxonomic biases in invasion ecology. Trends Ecol. Evol. 23: 237-44.

PYŠEK, P., V. JAROŠÍK, J. PERGL, L. MORAVCOVÁ, M. CHYTRÝ \& I. KÜHN. 2014. Temperate trees and shrubs as global invaders: the relationship between invasiveness and native distribution depends on biological traits. Biol. Invasions 16: 577-589.

PYŠEK, P. \& D. RICHARDSON. 2007. Traits associated with invasiveness in alien plants: where do we stand? In: NENTWIG W. (ed.), Biological Invasions. Ecological Studies Vol. 193, pp. 97-125. SpringerVerlag, Berlin. Heidelberg.

REICHARD, S. 2011. Horticulture. In: SIMBERLOFF D. \& M. REJMÁNEK (eds.), Encyclopedia of biological Invasions, pp. 336-342, University of California Press, Berkeley.

REJMÁNEK M. \& D. M. RICHARDSON 2013. Trees and shrubs as invasive alien species - 2013 update of the global database. Divers. Distrib. 19: 1-2.

REJMÁNEK, M. 2014. Invasive trees and shrubs: where do they come from and what we should expect in the future? Biol. Invasions 16: 483-498.

REJMÁNEK, M., D. M. RICHARDSON \& P. PYŠEK. 2005. Plant invasions and invisibility of plant communities. In: VAN DER MAAREL, E. (ed.), Vegetation Ecology, pp: 332-55. Blackwell, Oxford.

REJMÁNEK, M., D. M. RICHARDSON, S. I. HIGGINS, M. J. PITCAIRN \& E. GROTKOPP. 2005. Ecology of invasive plants - state of the art. Invasive alien species: a new synthesis. In: MOONEY, H. A., R. N.MACK, J. A. MC NEELY, L. NEVILLE, P.J. SCHEI \& J. WAAGE (eds.), Invasive alien species: searching for solutions, pp. 104-161, Island Press, Washington, D.C.

RICHARDSON D. M. \& M. REJMÁNEK. 2011. Trees and shrubs as invasive alien species - a global review. Divers. Distrib. 17: 788-809.

RICHARDSON, D. M., C. HUI, M. A. NUÑEZ, \& A. PAUCHARD. 2014. Tree invasions: patterns, processes, challenges and opportunities. Biol. Invasions 16: 473-481.

RICHARDSON, D. M., P. PYŠEK \& J. T. CARLTON. 2011. A compendium of essential concepts and terminology in invasion ecology. Fifty years of invasion ecology. In: RICHARDSON D.M. (ed.), The legacy of Charles Elton. pp. 409-420, WileyBlackwell, Oxford.
SAYAGO, M. 1969. Estudio fitogeográfico del norte de Córdoba. Bol. Academia Nac. Cienc. Córdoba 46: 123-427.

SPEZIALE K. L., S. A. LAMBERTUCCI, M. CARRETE \& J. L. TELLA. 2012. Dealing with non-native species: what makes the difference in South America? Biol. Invasions 14: 1609-1621.

SCOPUS ${ }^{\circledR}$, a product of Elsevier B.V., Radarweg 29, 1043NX Amsterdam, The Netherlands

TECCO P.A. 2006. Riqueza, abundancia y características morfo-funcionales de las especies nativas y exóticas establecidas bajo distintas condiciones de uso de la tierra en cinco ecosistemas del centro-oeste de Argentina. Tesis doctoral. Universidad Nacional de Córdoba. Argentina.

TECCO P. A., D. E. GURVICH, S. DÍAZ, N. PÉREZHARGUINDEGUY \& M. CABIDO M. 2006. Positive interaction between invasive plants: the influence of Pyracantha angustifolia on the recruitment of native and exotic woody species. Austral Ecol. 31: 293-300.

TECCO P. A., C. URCELAY, S. DÍAZ, M. CABIDO \& N. PÉREZ-HARGUINDEGUY. 2013. Contrasting functional trait syndromes underlay woody alien success in the same ecosystem. Austral Ecol. 38: 443-451.

VÁZQUEZ, D. \& R. ARAGÓN. 2002. Biological Invasions in southern South America: a First Step towards a Synthesis. Biol. Invasions 4: 1-2.

ZAK, M. \& M. CABIDO. 2004. Do subtropical seasonal forests in the Gran Chaco, Argentina, have a future? Biol. Conserv. 120: 589-598.

ZALBA, S. M. \& C. B. VILLAMIL. 2002. Woody plant invasion in relictual grasslands. Biol. Invasions 4: 55-72.

ZAVALETA, E.S. 2000. Valuing ecosystem services lost to Tamarix invasion in the United States. In: MOONEY H. A. \& R. J. HOBBS (eds.), Invasive species in a changing world, pp 261-300. Island Press, Washington, DC.

ZULOAGA, F.O., O. MORRONE \& M.J. BELGRANO. 2008. Catálogo de Plantas Vasculares del Cono Sur. Monogr. Missouri Bot. Garden (USA).

Recibido el 28 de abril de 2014, aceptado el 4 de noviembre de 2014 . 
Apéndice 1. Base de datos de los árboles y arbustos invasores la Provincia de Córdoba. Forma de Vida: A: árbol, Arb: arbustos. Género. Especie. Clado mayor (Clase). Orden. Familia. Nombre vulgar. Origen de las especies dentro de las siguientes áreas: Af: África; As: Asia; Au: Australia; E As: Este de Asia; Eu: Europa; ME: Medio oriente; N Af: Norte de África; NAm: Norte América; SAf: Sudáfrica; W As: Oeste de Asia. Exótica invasora o nativa invasora. Lugares invadidos del mundo: 1: invasora en dicha región (sensu Rejmánek \& Richardson 2013) o Nueva: se incorpora como nueva región invadida (no estaba incluida en Rejmánek \& Richardson 2013). Tipo de ambiente invadido en la Provincia de Córdoba, todos: presente simultáneamente en Bosques, matorrales, pastizales y bordes de río. Modo de dispersión. Usos o Motivos de introducción. Comparación con otras bases de datos. Incluidas en la Base mundial (1) o (nueva) se incorpora como nueva invasora a nivel global (no estaba incluida en Rejmánek \& Richardson 2013). Incluidas en I3N (1) o (nueva) se incorpora como nueva invasora en la base I3N o $\left(^{* *}\right)$ no corresponde por tratarse de una invasora nativa. Registradas en Córdoba (Catálogo) (1) o (nueva): se incorpora como nueva invasora en la provincia de Córdoba $\circ\left(^{* *}\right)$ no corresponde por tratarse de una invasora nativa. Registradas en Argentina (Catálogo): BA: Buenos Aires; $\mathrm{CH}$ : Chaco; $\mathrm{CHU}$ : Chubut; $\mathrm{CO}$ : Córdoba; COR: Corrientes; DF: Distrito Federal; ER: Entre Ríos; JU: Jujuy; LP: La pampa; MI: Misiones; NE: Neuquén; RN: Rio Negro; SA: Salta; SDE: Sgo. del Estero; SF: Santa Fe; TU: Tucumán, o (nueva) nuevo registro a incorporar en la base Catálogo o $\left({ }^{* *}\right)$ no corresponde por tratarse de una invasora nativa. Registradas en otros países (Catálogo) se completa con el nombre del país correspondiente, o $\left({ }^{* *}\right)$ no corresponde por tratarse de una invasora nativa (Sudamericana) o (ninguno): no se encuentra en otros países (sensu Catálogo) o (sin dato): es una especie que no se encontraba incorporada en el Catálogo. STATUS sensu Catálogo, se completa con la categoría correspondiente según esta base o (nueva): especie nueva a incorporar en la base Catálogo, con el status de INVASORA.

Database of the invasive exotic trees and shrubs of the Cordoba Province (Argentina). In bold the different information detailed in the data base. Life form: A: trees, Arb: shrubs. Genus. Species. Larger clade (Class). Order. Family. Common Name. Specie Origin within the following regions: Af: Africa; As: Asia; Au: Australia; E As: Eastern Asia; Eu: Europe; ME: Middle East; N Af: North Africa; NAm: North America; SAf: South Africa; W As: West Asia. Exotic invader or Native invader. Invaded regions of the world: 1: when recorded as invader in each region (sensu Rejmánek \& Richardson 2013) or new: when recorded as a new invaded region (i.e., not included in Rejmánek \& Richardson 2013). Invaded environment in Cordoba province. All: when present simultaneously in woodlands, shrublands, grasslands and river edges. Dispersal mode. Uses or reasons for introduction. Comparison with other bases. Included in the global base (1) or (new) when incorporated as a new invader globaly (i.e., not included in Rejmánek \& Richardson 2013). Included in I3N (1) or (new) when incorporated as a new invader in the I3N base or $\left({ }^{* *}\right)$ when not applicable because it is a native invader. Included in Córdoba (Catalog) (1) or (new) when incorporated as a new invader in the Córdoba province or $\left({ }^{* *}\right)$ when not applicable because it is a native invader. Included in Argentina (Catalog): different argentinean province indicated as BA: Buenos Aires; CH: Chaco; CHU: Chubut; CO: Córdoba; COR: Corrientes; DF: Distrito Federal; ER: Entre Ríos; JU: Jujuy; LP: La Pampa; MI: Misiones; NE: Neuquén; RN: Rio Negro; SA: Salta; SDE: Sgo. del Estero; SF: Santa Fe; TU: Tucumán, or (new): when incorporated as a new record in the Catalog base or $\left(^{* *}\right)$ when not applicable because it is a native invader. Included in other countries (Catalog), completed with the country names or $\left(^{* *}\right)$ when not applicable because it is a native invader (Southamerica) or (none): when it is not recorded in other countries (sensu Catalog) or (without data): when a specie not included in Catalog. STATUS sensu Catalog, completed with catalog's information or (new): when it is new specie for Catalog with the INVASIVE's status.

Esta base de datos puede ser descargada en formato excell desde el sitio web de la revista. This database can be downloaded in excell format from the web site of the journal. 


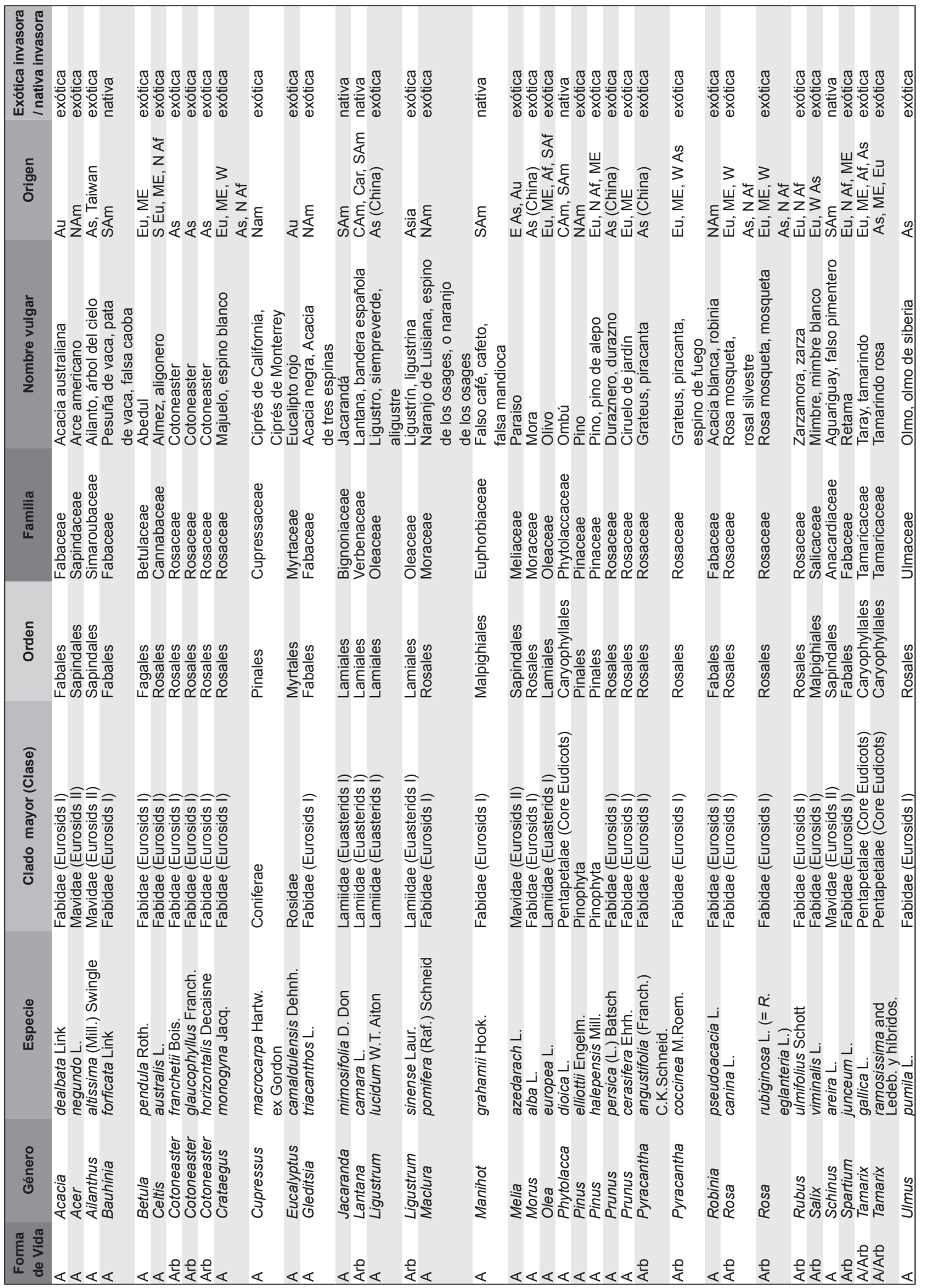


Bol. Soc. Argent. Bot. 49 (4) 2014

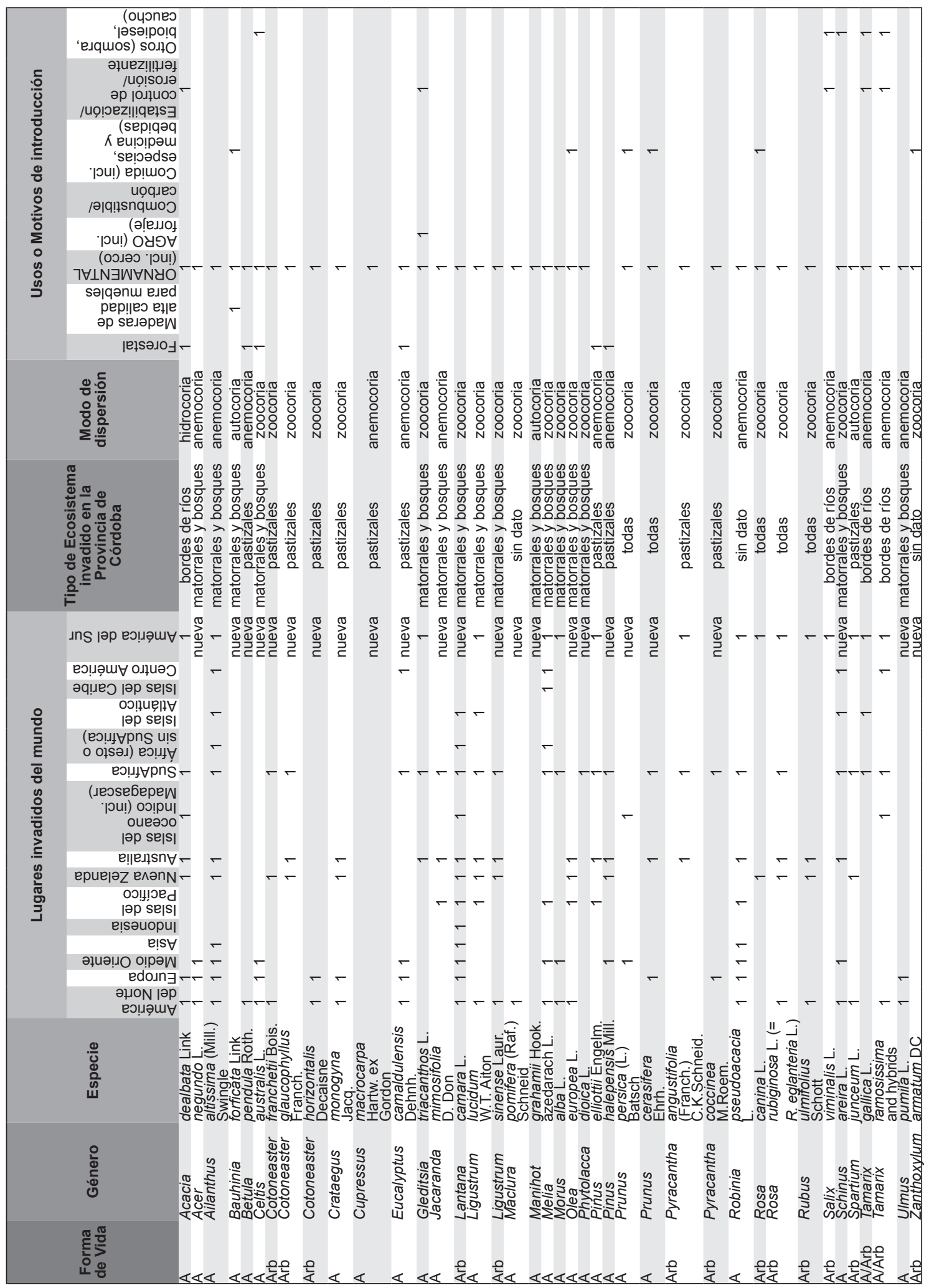




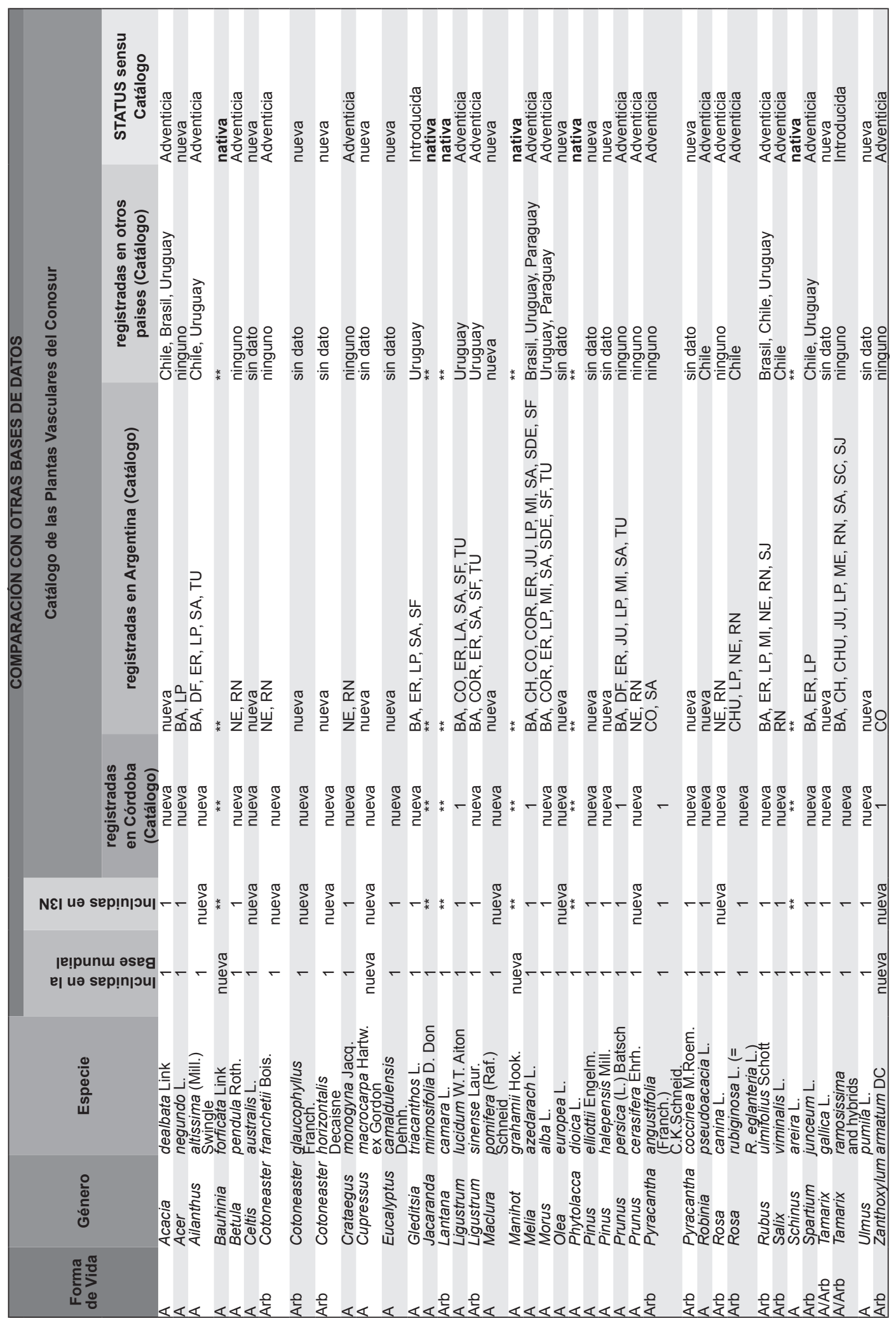




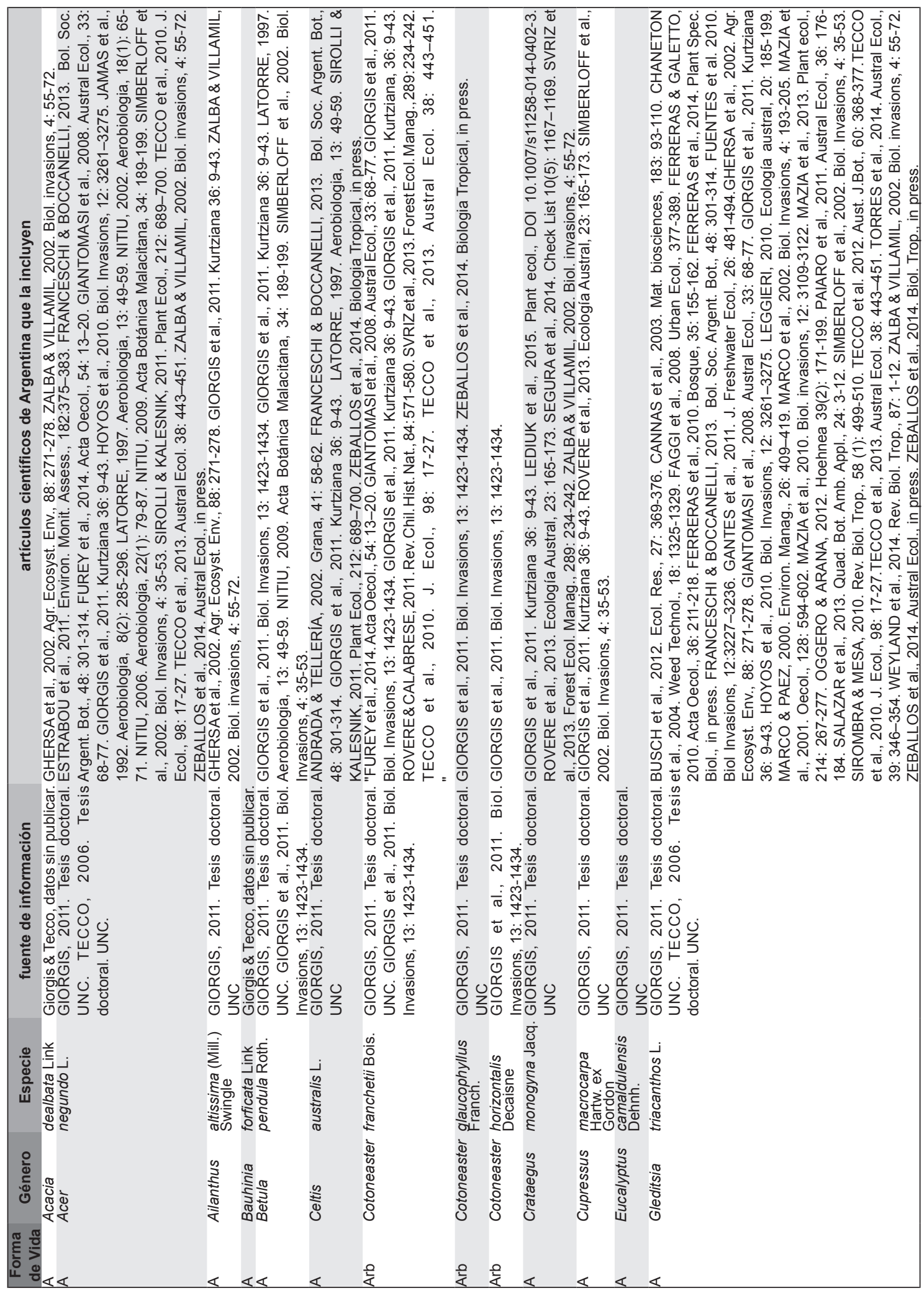




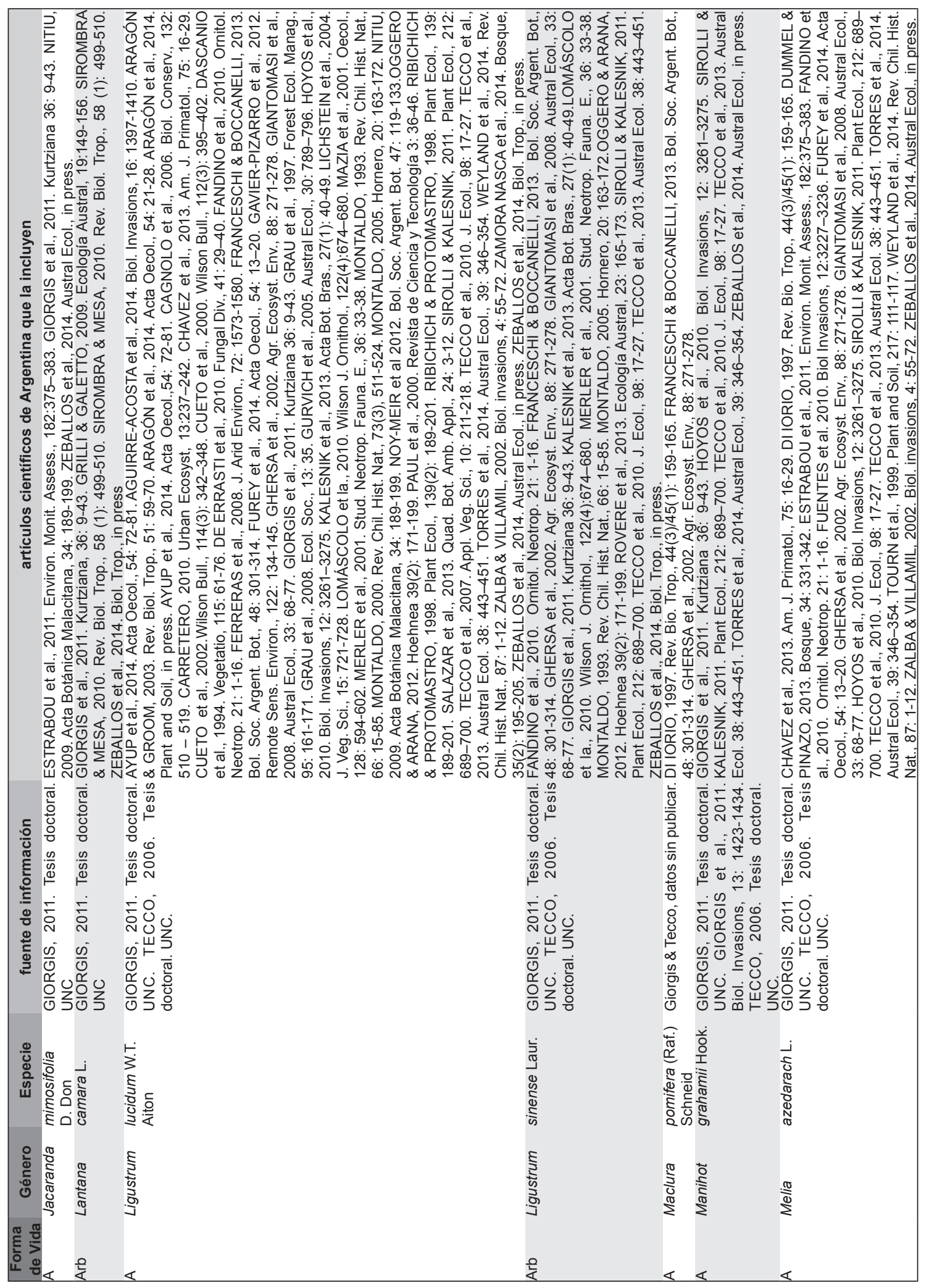




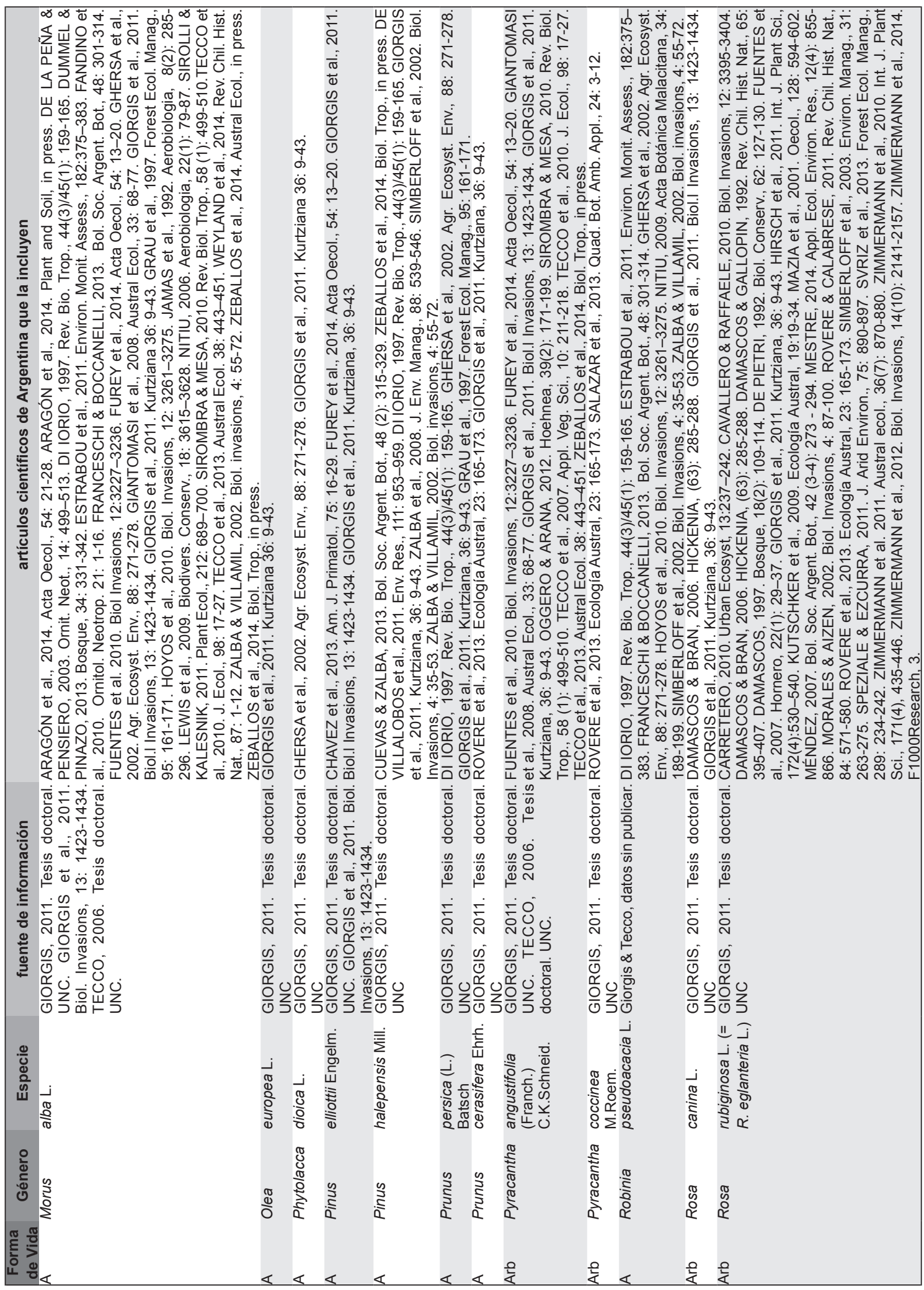


M. A. Giorgis y P. A. Tecco - Leñosas invasoras de Córdoba

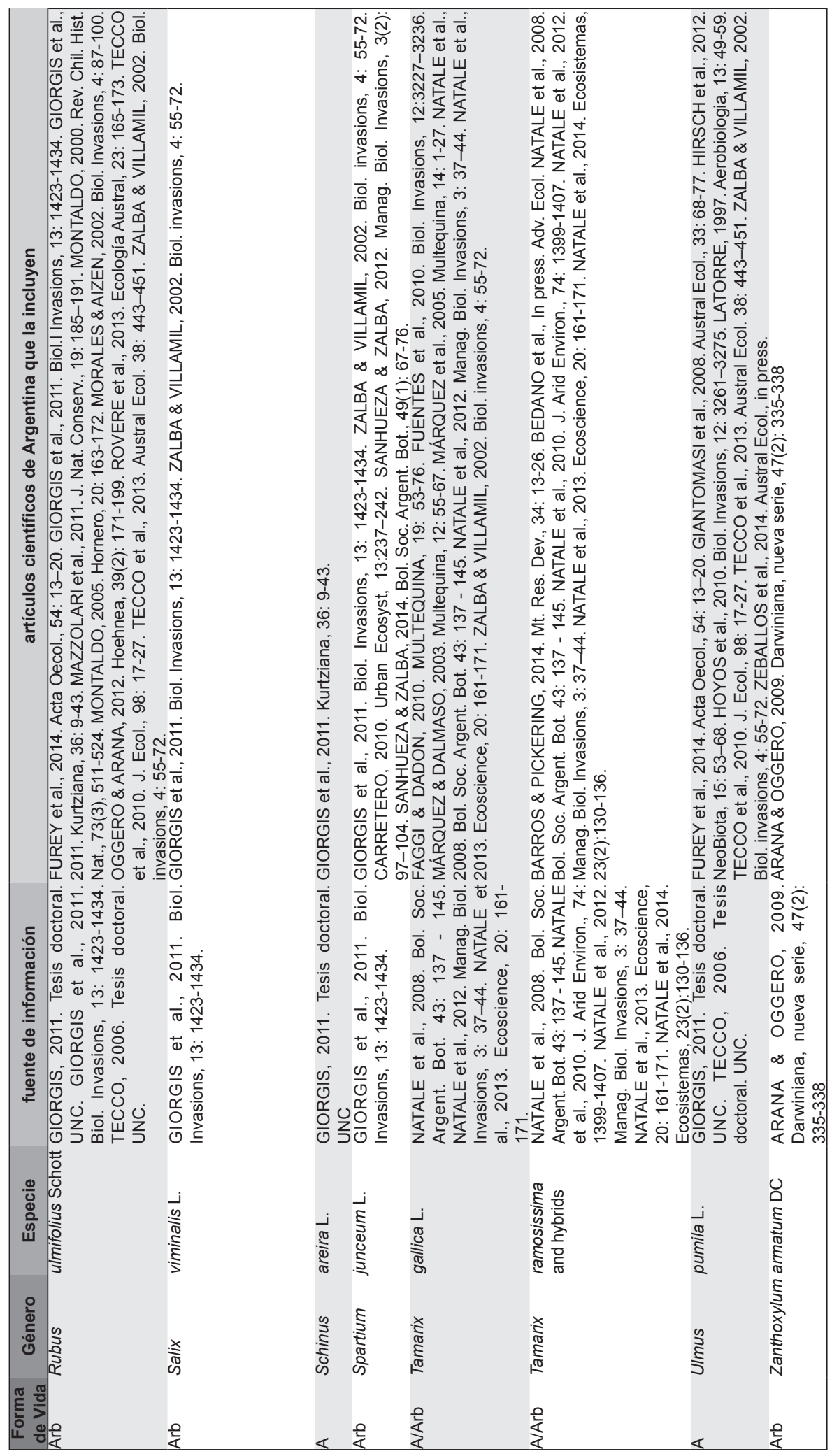


